\title{
Multiscale modeling of crowdion and vacancy defects in body-centered-cubic transition metals
}

\author{
P. M. Derlet, ${ }^{1}$ D. Nguyen-Manh, ${ }^{2}$ and S. L. Dudarev ${ }^{2,3}$ \\ ${ }^{1}$ Paul Scherrer Institute, CH-5232 Villigen PSI, Switzerland \\ ${ }^{2}$ EURATOM/UKAEA Fusion Association, Culham Science Centre, Oxfordshire OX14 3DB, United Kingdom \\ ${ }^{3}$ Department of Physics, Imperial College, Exhibition Road, London SW7 2AZ, United Kingdom \\ (Received 11 October 2006; revised manuscript received 21 March 2007; published 6 August 2007)
}

\begin{abstract}
We investigate the structure and mobility of single self-interstitial atom and vacancy defects in bodycentered-cubic transition metals forming groups 5B (vanadium, niobium, and tantalum) and 6B (chromium, molybdenum, and tungsten) of the Periodic Table. Density-functional calculations show that in all these metals the axially symmetric $\langle 111\rangle$ self-interstitial atom configuration has the lowest formation energy. In chromium, the difference between the energies of the $\langle 111\rangle$ and the $\langle 110\rangle$ self-interstitial configurations is very small, making the two structures almost degenerate. Local densities of states for the atoms forming the core of crowdion configurations exhibit systematic widening of the "local" $d$ band and an upward shift of the antibonding peak. Using the information provided by electronic structure calculations, we derive a family of Finnis-Sinclair-type interatomic potentials for vanadium, niobium, tantalum, molybdenum, and tungsten. Using these potentials, we investigate the thermally activated migration of self-interstitial atom defects in tungsten. We rationalize the results of simulations using analytical solutions of the multistring Frenkel-Kontorova model describing nonlinear elastic interactions between a defect and phonon excitations. We find that the discreteness of the crystal lattice plays a dominant part in the picture of mobility of defects. We are also able to explain the origin of the non-Arrhenius diffusion of crowdions and to show that at elevated temperatures the diffusion coefficient varies linearly as a function of absolute temperature.
\end{abstract}

DOI: 10.1103/PhysRevB.76.054107 PACS number(s): 61.72.Bb, 61.80.Az, 63.20.Mt, 66.30. $-\mathrm{h}$

\section{INTRODUCTION}

The fact that in the body-centered-cubic (bcc) lattice the energetically favorable interstitial position is not the geometrically obvious center of a face of the cubic unit cell but rather a position between the nearest neighbors was first noted by Paneth ${ }^{1}$ in connection with the study of selfinterstitial atom configurations in bcc alkali metals. Paneth introduced the term "crowdion" to describe the extended distortion of the crystal lattice in the 111 direction, resulting from the embedding of an extra atom in the lattice. Figure 1 shows schematic models of several defects, including a 111 crowdion, in the bcc lattice. The interstitial defect structures, shown in darker color in Fig. 1, result from the embedding of just one extra atom in the lattice, followed by the elastic relaxation of positions of all the atoms in the region surrounding the defect.

The crowdion concept proposed by Paneth ${ }^{1}$ was further extended to the case of fcc metals by Lomer and Cottrell, ${ }^{2}$ who discussed annealing of point defects in metals and alloys. This concept was then further developed by Blewitt $e t$ $a l .{ }^{3}$ and by Tewordt. ${ }^{4}$ de Wette investigated the formation of crowdions in the Wigner electron lattices. ${ }^{5}$ Frank and Seeger used the crowdion concept in the formulation of the so-called two-interstitial model of radiation damage, ${ }^{6}$ initiating a period of extensive debate in the literature about the structure of self-interstitial configurations. Seeger and $\mathrm{Chik}^{7}$ investigated the possible occurrence of an extended interstitial configuration, stabilized by phonon entropy, in silicon. Their idea received further attention in connection with the problem of self-diffusion in silicon, ${ }^{8,9}$ where experimental observations showed that self-interstitial atoms were able to migrate at extremely low temperatures of the order of several kelvins.
The fact that the axially symmetric "one-dimensional" structure of a crowdion configuration was intimately related to its high one-dimensional mobility was noted by Seeger. ${ }^{10}$ Schilling et al. ${ }^{11}$ disagreed with the crowdion hypothesis and argued that the three-dimensional model of diffusion of defects gave better agreement with experimental observations of defect migration in fcc copper. Frank ${ }^{12}$ noted that the isochronal low-temperature recovery experiments in niobium provided evidence for the formation of highly mobile crowdion configurations under irradiation. Extending this idea, Frank and Seeger ${ }^{13}$ proposed that the low-temperature migration of self-interstitial atom defects in bcc metals could be explained in terms of diffusion of crowdions. Woo and Frank $^{14}$ used the crowdion diffusion model to explain the origin of void lattices forming in some metals under irradiation. Indenbom ${ }^{15}$ examined the part played by crowdions in the picture of plastic deformation of materials. His theoretical ideas were corroborated by experiments carried out in bcc tungsten. ${ }^{16,17}$ High mobility of self-interstitial atom defects in tungsten at low temperatures was observed experimentally in Ref. 18.

At the same time, the accumulation of experimental information on thermally activated mobility of defects in bcc metals did not explicitly address the question about the structure of these defects. Some experimental evidence (for example, the high mobility of defects at temperatures below $40 \mathrm{~K} \mathrm{ob-}$ served in all the nonmagnetic bcc metals ${ }^{19,20}$ ) pointed to the possible role played by the highly mobile $\langle 111\rangle$ crowdions, while other observations ${ }^{21}$ remained at variance with the crowdion model. Atomistic simulations based on semiempirical potentials appeared equally inconclusive, with some simulations predicting the $\langle 110\rangle$ dumbbell while others predicting the $\langle 111\rangle$ crowdion as the lowest-energy configuration of a self-interstitial atom defect. ${ }^{22-26}$ 

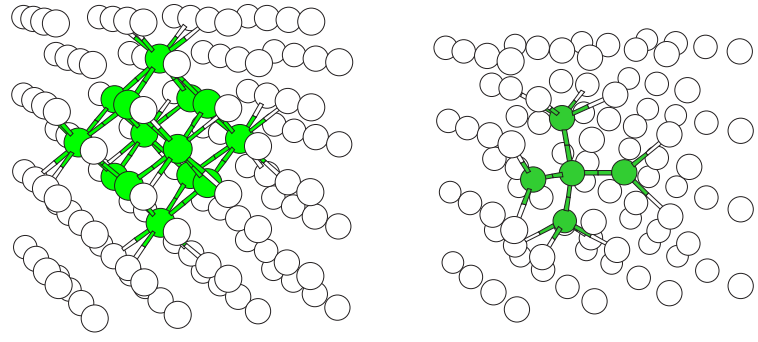

vacancy

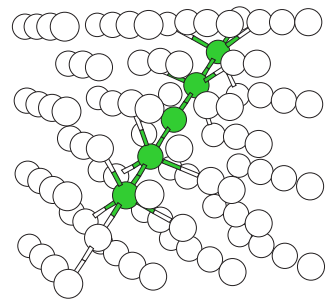

111

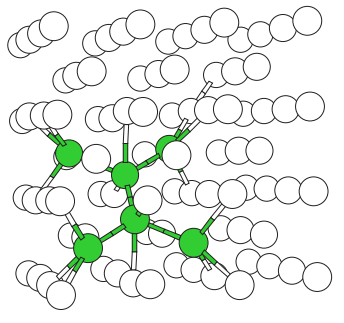

110 tetrahedral

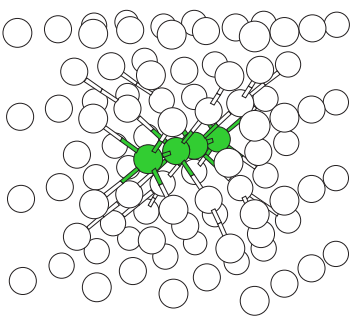

100

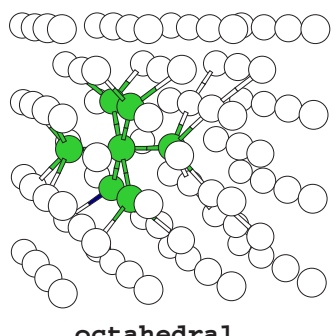

octahedral

FIG. 1. (Color online) Schematic view of the vacancy and the five high-symmetry self-interstitial atom configurations in a bcc metal. A 111 crowdion configuration shown in this figure is formed by inserting an extra atom halfway between the nearest neighbors and by relaxing the resulting configuration at $T=0$. A tetrahedral configuration is formed if an interstitial atom is placed at $(a / 4, a / 2,0)$, where $a$ is the parameter of the crystal lattice. An octahedral configuration is formed if an extra atom is placed at the center of the face of the cell, for example, at $(a / 2, a / 2,0)$.

Large-scale density-functional calculations performed in the last several years provided significantly more reliable and largely free from model assumptions information about the energies of formation of stable, as well as metastable, configurations of self-interstitial atom defects. These configurations, illustrated in Fig. 1, have different formation energies, and the first question that one has to address before proceeding to the investigation of dynamical properties of defects, for example, their thermally activated migration or migration driven by external stress fields, is the question about the relative energies of defect structures shown in Fig. 1.

Density-functional calculations ${ }^{27-29}$ showed that in bcc Fe a self-interstitial atom adopts the $\langle 110\rangle$ configuration, while in vanadium and molybdenum the lowest-energy configuration of a defect has the $\langle 111\rangle$ symmetry. ${ }^{30} \mathrm{~A}$ recent study of point defects spanning the entire group of bcc metals ${ }^{31}$ confirmed that the $\langle 111\rangle$ crowdion configuration does, indeed, have the lowest formation energy in all the nonmagnetic bcc metals except chromium, where the difference between the energies of the $\langle 111\rangle$ and $\langle 110\rangle$ configurations is very small.

An unusual feature of the crowdion configuration is that the displacements of atoms in it are effectively one dimensional and that the strain field in the string containing a selfinterstitial atom can be accurately described by an analytically tractable Frenkel-Kontorova model. ${ }^{32}$ This model also applies to the treatment of the elastic field of the crowdion defect, ${ }^{33}$ making it possible to derive equations of motion for crowdions in the lattice. ${ }^{34}$ The multi-string FrenkelKontorova model, describing clusters of interstitial atoms, shows that there is a link between the soliton solutions for a crowdion and for an edge dislocation. ${ }^{35}$ Furthermore, the fact that in the continuum limit the strain field of a crowdion is well approximated by the sine-Gordon equation ${ }^{36,37}$ makes the latter a convenient model for studying the Brownian motion of defects at elevated temperatures. Migration of crowdion defects in bcc vanadium was recently investigated using molecular dynamics. ${ }^{38}$ Molecular dynamics simulations also showed that small clusters of self-interstitial atom defects have properties similar to those of crowdion configurations, including their high thermally activated one-dimensional mobility. ${ }^{39-45}$

The main objective of this paper is to perform a comprehensive multiscale investigation of properties of crowdion defects in bcc metals. We start from giving a detailed account of electronic structure and energies of formation of selfinterstitial and vacancy defects for the entire group of bcc metals. On the basis of these calculations, we derive a family of semiempirical potentials of the Finnis-Sinclair type for vanadium, niobium, tantalum, molybdenum, and tungsten. We test these potentials by simulating structures that were not included in the fitting procedure and find a generally good agreement with density-functional calculations. We also find that the magnitude of the bonding part of the potential for each metal is well correlated with the respective second moment of the density of $d$ states. Furthermore, we observe that the concept of local variation of the width of the $d$ band underlying the Finnis-Sinclair functional form of the potentials is well correlated with the results of electronic structure calculations that exhibit local broadening of the $d$ band near the core of the defects.

Using the developed potentials, we extend the treatment to longer time and larger spatial scales and investigate dynamical properties of the $\langle 111\rangle$ crowdions and their migration at elevated temperatures. An analysis of the migration of crowdions in tungsten at temperatures higher than the Debye temperature of the material shows, in agreement with a similar recent study of defects in vanadium, ${ }^{38}$ that the Brownian motion of crowdions does not follow the Arrhenius law and that the diffusion coefficient of a defect varies approximately linearly as a function of absolute temperature. We rationalize this observation using an analytically solvable FrenkelKontorova model that describes a nonlinear elastic interaction between a crowdion and the field of phonon excitations in the lattice. We find that the linear variation of the diffusion coefficient, as a function of absolute temperature observed in simulations, is related to the fact that the crystal lattice, where the crowdion migrates, is discrete. An analysis of the statistics of the $\langle 111\rangle$ to $\langle 110\rangle$ transformations of the crowdion defect shows that the longitudinal Brownian motion of a crowdion is fairly stable and that rotational $\langle 111\rangle \leftrightarrow\langle 110\rangle$ 
transformations have a significant effect on the diffusion of crowdions only in the high-temperature limit.

We conclude our study by reviewing the multiscale aspect of the problem, including the pivotal role played by the analysis of the electronic structure of defects, the use of controlled approximations in deriving the semiempirical potentials, and the essential part played by solvable analytical models in rationalizing the information provided by atomistic simulations.

\section{ELECTRONIC STRUCTURE OF DEFECTS FROM DENSITY FUNCTIONAL THEORY CALCULATIONS}

The total-energy and electronic structure calculations for nonmagnetic bcc transition metals described in this section were performed using density-functional theory (DFT) within the generalized gradient approximation (GGA) implemented in the package of linear combination of atomic type orbitals (PLATO). ${ }^{46}$ This code has recently undergone major development to allow modeling of defects ${ }^{31}$ and $a b$ initio molecular dynamic simulations. ${ }^{47}$ Since our previous communication $^{31}$ provided only a brief description of the method, below, we describe the significant technical aspects of our calculations.

PLATO uses strictly localized atomiclike basis functions. These are found by solving for an atom in an external spherical potential well $U(r)$, where the potential $U(r)$ is assumed to diverge for $r>r_{c}$, where $r_{c}$ is a user-defined cutoff radius. This condition results in a set of basis functions vanishing at $r=r_{c}$ and retaining nonvanishing first and second derivatives at that point. To ensure that these derivatives vanish at $r_{c}$, the functions are multiplied by the factor $1-\exp [-(r$ $\left.\left.-r_{c}\right)^{2} /\left(2 \sigma^{2}\right)\right]$, where $\sigma$ is an adjustable parameter. Basis sets are constructed by using functions from both an atom and a positively charged ion solved in this way. Polarization functions are also included in the basis set since they play an important part in giving the basis set the required variational freedom. Basis functions having the same angular form are orthogonalized since this improves the stability of calculations. Basis sets consisting of two sets of functions corresponding to a neutral atom and one set of functions corresponding to a doubly ionized atom, referred to as the double numeric plus polarization (DNP) basis, are used in most cases described below.

To achieve a high accuracy in the DFT calculation of defect structures in transition metals, treating both valence and semicore electrons becomes equally important. Therefore, these electrons have been included in the construction of the DNP basis set consisting of 18 atomiclike functions per atom. For the $n d(n=3,4,5)$ transition metal series, we retain the $n s, n p, n d$, and $(n+1) s$ orbitals for the neutral atom and the $n d^{*},(n+1) s^{*}$, and $(n+1) p^{*}$ orbitals for the doubly ionized atom. All the orbitals are cut off at the critical radius $r_{c}$ of 8.0 a.u., and their tails are smoothed over a distance $\sigma$ of about 1.5 a.u. The calculations were performed using the relativistic semicore separable pseudopotentials developed by Hartwigsen et al. ${ }^{48}$ with the Perdew-Burke-Ernzerhof $(\mathrm{PBE})^{49}$ GGA for the exchange and correlation functional. The fast Fourier transform (FFT) mesh was used to evaluate numerical integrals with atomic orbitals and to solve Poisson's equation. The electron charge density was projected onto a uniform FFT mesh with spacing of 0.2 a.u. A denser grid of 0.15 a.u. was used to assess the precision of the charge density calculations. This, in turn, was used to estimate the systematic error of our defect structure total-energy calculations, which we found to be of the order of $0.01 \mathrm{eV}$. Both the volume and ionic relaxations were used in the PLATO calculations, and the forces and stress components in relaxed configurations were smaller than $0.025 \mathrm{eV} / \AA$ and $0.001 \mathrm{Mbar}$, respectively. All the calculations of defect structures were performed using a reference bcc supercell containing 128 atoms and a $3 \times 3 \times 3$ shifted $k$-point grid. In the case of $\mathrm{Cr}$, we assumed the nonmagnetic ground state, the total energy of which is very close to the energy of the antiferromagnetic state found in recent full-potential linearized augmented plane wave calculations..$^{50}$ For completeness, $a b$ initio calculations were also performed for ferromagnetic $\alpha$-Fe where we used a pseudopotential generalized by the projector augmented wave (PAW) approach and implemented in the Vienna $a b$ initio simulation package (VASP)..$^{51-53}$ This pseudopotential, which includes the effect of semicore electrons, was taken from the VASP library. Spinpolarized calculations were performed using the GGA-PBE exchange-correlation (XC) functional. The plane-wave cutoff energy of $300 \mathrm{eV}$ and $27 k$ points were used for the $128+1$ atom supercells.

Table I gives the calculated formation energies of the vacancy and interstitial atom defects. ${ }^{31,54,55}$ These energies are used for fitting interatomic potentials described in the next section of the paper. Our DFT study shows that the $\langle 111\rangle$ type defect configurations have the lowest formation energies in all the nonmagnetic bcc metals. The energy difference between the $\langle 110\rangle$ and the $\langle 111\rangle$ configurations is smaller in group $6 \mathrm{~B}$ than in group $5 \mathrm{~B}$. The pattern of ordering of selfinterstitial atom (SIA) configurations in the nonmagnetic bcc metals is fundamentally different from that of ferromagnetic bcc Fe, where the $\langle 110\rangle$ dumbbell has the lowest formation energy ${ }^{28,59}$ found in SIESTA (GGA-PBE) and VASP [GGA with the Perdew-Wang (PW) XC functional ${ }^{61}$ ] calculations. This is also confirmed by the present VASP (GGA-PBE) calculations. We also find that apart from the significant differences between the energies of the $\langle 110\rangle$ and $\langle 111\rangle$ configurations, all the other SIA configurations in Fe follow the same relative pattern of ordering of formation energies as the SIA configurations in groups $5 \mathrm{~B}$ and $6 \mathrm{~B}$; i.e., the formation energies increase from the $\langle 111\rangle$ dumbbell to the $\langle 110\rangle$, the tetrahedral, the $\langle 100\rangle$ dumbbell, and to the octahedral configurations.

Defects follow a systematic pattern not only for the case of SIAs but also for the case of monovacancy formation and migration energies. ${ }^{31,54,55}$ These energies were calculated using the nudged elastic band method, ${ }^{62}$ and results agree well with the available experimental data. ${ }^{20}$ Experimentally measured values for the vacancy migration energy in molybdenum were reported by several groups. Measurements performed by the Delft group, where helium desorption was used for determining vacancy concentration, gave the value of $1.23 \pm 0.05 \mathrm{eV} .{ }^{63}$ This value is similar to the value corresponding to the stage III recovery of electron irradiated mo- 
TABLE I. The first four rows of this table give basic parameters of all the bcc transition metals of groups $5 \mathrm{~B}$ and $6 \mathrm{~B}$, and those for Fe (group 8) evaluated using DFT, and compare them with the experimentally measured values taken from Refs. 56 and 20. The remaining rows of the table give defect formation $(f)$, migration $(m)$, and binding $(b)$ energies (in $\mathrm{eV}$ units). Values referring to the interstitial configurations $(\langle 111\rangle$ dumbbell, etc.) give the formation energies of the corresponding defect structures.

\begin{tabular}{|c|c|c|c|c|c|c|c|}
\hline & V & $\mathrm{Nb}$ & $\mathrm{Ta}$ & $\mathrm{Cr}$ & Mo & W & $\mathrm{Fe}$ \\
\hline \multirow[t]{2}{*}{$a(\AA)$} & $3.04^{\mathrm{a}}$ & $3.32^{\mathrm{a}}$ & $3.31^{\mathrm{a}}$ & $2.85^{\mathrm{a}}$ & $3.17^{\mathrm{a}}$ & $3.18^{\mathrm{a}}$ & $2.84^{\mathrm{b}}$ \\
\hline & $3.03^{\mathrm{c}}$ & $3.30^{\mathrm{c}}$ & $3.30^{\mathrm{c}}$ & $2.88^{\mathrm{c}}$ & $3.15^{\mathrm{c}}$ & $3.16^{\mathrm{c}}$ & $2.87^{\mathrm{c}}$ \\
\hline \multirow[t]{2}{*}{$B$ (Mbar) } & $1.71^{\mathrm{a}}$ & ${ }^{\mathrm{a}} 1.73$ & $1.99^{\mathrm{a}}$ & $1.92^{\mathrm{a}}$ & $2.68^{\mathrm{a}}$ & $3.05^{\mathrm{a}}$ & $1.82^{\mathrm{b}}$ \\
\hline & $1.62^{\mathrm{c}}$ & $1.70^{\mathrm{c}}$ & $2.00^{\mathrm{c}}$ & $1.90^{\mathrm{c}}$ & $2.72^{\mathrm{c}}$ & $3.23^{\mathrm{c}}$ & $1.68^{\mathrm{c}}$ \\
\hline \multirow[t]{2}{*}{$H_{f}^{v}$} & $2.51^{\mathrm{a}}$ & $2.99^{a}$ & $3.14^{\mathrm{a}}$ & $2.64^{\mathrm{a}}$ & $2.96^{\mathrm{a}}$ & $3.56^{\mathrm{a}}$ & $2.15^{\mathrm{a}}$ \\
\hline & $2.1-2.2^{\mathrm{d}}$ & $2.6-3.1^{\mathrm{d}}$ & $2.8-3.1^{\mathrm{d}}$ & $2.0-2.4^{\mathrm{d}}$ & $2.6-3.2^{\mathrm{d}}$ & $3.5-4.1^{\mathrm{d}}$ & $1.6-2.2^{\mathrm{d}}$ \\
\hline \multirow[t]{2}{*}{$H_{m}^{v}$} & $0.62^{\mathrm{a}}$ & $0.91^{\mathrm{a}}$ & $1.48^{\mathrm{a}}$ & $0.91^{\mathrm{a}}$ & $1.28^{\mathrm{a}}$ & $1.78^{\mathrm{a}}$ & $0.64^{\mathrm{b}}, 0.67^{\mathrm{e}}$ \\
\hline & $0.5-1.2^{\mathrm{d}}$ & $0.6-1.0^{\mathrm{d}}$ & $0.7-1.9^{\mathrm{d}}$ & $0.95^{\mathrm{d}}$ & $1.3-1.6^{\mathrm{d}}$ & $1.7-2.0^{\mathrm{d}}$ & $0.55^{\mathrm{d}}$ \\
\hline \multirow[t]{2}{*}{$H_{f}^{2 v}(1 N N)$} & $4.94^{\mathrm{f}}$ & $5.62^{\mathrm{f}}$ & $6.01^{\mathrm{f}}$ & $5.01^{\mathrm{f}}$ & $5.60^{\mathrm{f}}$ & $6.71^{\mathrm{f}}$ & $4.08^{\mathrm{b}}$ \\
\hline & $3.90-3.99^{\mathrm{g}}$ & $5.11^{\mathrm{g}}$ & $5.47^{\mathrm{g}}$ & $3.34^{\mathrm{g}}$ & $5.75^{\mathrm{g}}$ & $7.32^{\mathrm{g}}$ & $3.31^{\mathrm{g}}, 3.85^{\mathrm{h}}$ \\
\hline \multirow[t]{2}{*}{$H_{f}^{2 v}(2 N N)$} & $4.80^{\mathrm{f}}$ & $5.57^{\mathrm{f}}$ & $5.89^{\mathrm{f}}$ & $5.16^{\mathrm{f}}$ & $5.78^{f}$ & $6.93^{\mathrm{f}}$ & $4.01^{\mathrm{b}}$ \\
\hline & $3.90-4.21^{\mathrm{g}}$ & $5.11^{\mathrm{g}}$ & $5.50^{\mathrm{g}}$ & $3.35^{\mathrm{g}}$ & $5.77^{\mathrm{g}}$ & $7.36^{\mathrm{g}}$ & $3.34^{\mathrm{g}}, 3.83^{\mathrm{h}}$ \\
\hline \multirow[t]{2}{*}{$H_{b}^{2 v}(1 N N)$} & $0.08^{f}$ & $0.36^{f}$ & $0.27^{f}$ & $0.27^{\mathrm{f}}$ & $0.32^{f}$ & $0.41^{\mathrm{f}}$ & $0.22^{\mathrm{b}}$ \\
\hline & $0.30-0.45^{\mathrm{g}}$ & $0.39^{\mathrm{g}}$ & $0.43^{\mathrm{g}}$ & $0.26^{\mathrm{g}}$ & $0.45^{\mathrm{g}}, 0.39^{\mathrm{i}}$ & $0.58^{\mathrm{g}}, 0.45^{\mathrm{i}}$ & $0.27^{\mathrm{g}}, 0.22,{ }^{\mathrm{i}} 0.19^{\mathrm{h}}$ \\
\hline \multirow[t]{2}{*}{$H_{b}^{2 v}(2 N N)$} & $0.22^{\mathrm{f}}$ & $0.41^{\mathrm{f}}$ & $0.39^{f}$ & $0.12^{\mathrm{f}}$ & $0.14^{\mathrm{f}}$ & $0.19^{f}$ & 0.29 \\
\hline & $0.30-0.23^{\mathrm{g}}$ & $0.39^{\mathrm{g}}$ & $0.41^{\mathrm{g}}$ & $0.25^{\mathrm{g}}$ & $0.43^{\mathrm{g}}, 0.25^{\mathrm{i}}$ & $0.54^{\mathrm{g}}, 0.29^{\mathrm{i}}$ & $0.24^{\mathrm{g}}, 0.15^{\mathrm{i}}, 0.30^{\mathrm{e}}, 0.21^{\mathrm{h}}$ \\
\hline \multirow[t]{2}{*}{$\langle 111\rangle$ dumbbell } & $3.367^{\mathrm{a}}$ & $5.253^{\mathrm{a}}$ & $5.832^{\mathrm{a}}$ & $5.685^{\mathrm{a}}$ & $7.417^{\mathrm{a}}$ & $9.548^{\mathrm{a}}$ & $4.61^{b}$ \\
\hline & $3.14^{\mathrm{j}}$ & $4.795^{\mathrm{k}}$ & $7.157^{1}, 5.858^{\mathrm{i}}$ & $5.68^{\mathrm{m}}$ & $7.34^{\mathrm{j}}$ & $8.919^{k}$ & $4.72,{ }^{\mathrm{m}} 4.34^{\mathrm{e}}$ \\
\hline \multirow[t]{2}{*}{$\langle 111\rangle$ crowdion } & $3.371^{\mathrm{a}}$ & $5.254^{\mathrm{a}}$ & $5.836^{\mathrm{a}}$ & $5.660^{\mathrm{a}}$ & $7.419^{\mathrm{a}}$ & $9.551^{\mathrm{a}}$ & $4.64^{\mathrm{b}}$ \\
\hline & $3.15^{\mathrm{h}}$ & $4.857^{\mathrm{k}}$ & $7.158^{\mathrm{k}}, 5.859^{\mathrm{l}}$ & & $7.34^{\mathrm{i}}$ & $8.893^{\mathrm{k}}$ & \\
\hline \multirow[t]{2}{*}{$\langle 110\rangle$ dumbbell } & $3.652^{\mathrm{a}}$ & $5.597^{\mathrm{a}}$ & $6.382^{\mathrm{a}}$ & $5.674^{\mathrm{b}}$ & $7.581^{\mathrm{a}}$ & $9.844^{\mathrm{a}}$ & $3.93^{b}$ \\
\hline & $3.48^{\mathrm{j}}$ & $4.482^{\mathrm{k}}$ & $6.847^{\mathrm{k}}, 6.557^{\mathrm{l}}$ & $5.66^{\mathrm{m}}$ & $7.51^{\mathrm{j}}$ & $9.641^{\mathrm{k}}$ & $4.03^{\mathrm{m}}, 3.64^{\mathrm{e}}$ \\
\hline \multirow[t]{2}{*}{ Tetrahedral } & $3.835^{\mathrm{a}}$ & $5.758^{\mathrm{a}}$ & $6.771^{\mathrm{a}}$ & $6.189^{\mathrm{a}}$ & $8.401^{\mathrm{a}}$ & $11.05^{\mathrm{a}}$ & $4.32^{\mathrm{b}}$ \\
\hline & $3.69^{\mathrm{j}}$ & & $6.845^{1}$ & & $8.20^{\mathrm{j}}$ & & $4.43,{ }^{\mathrm{m}} 4.26^{\mathrm{e}}$ \\
\hline \multirow[t]{2}{*}{$\langle 100\rangle$ dumbbell } & $3.918^{\mathrm{a}}$ & $5.949^{\mathrm{a}}$ & $7.003^{\mathrm{a}}$ & $6.643^{\mathrm{a}}$ & $9.004^{\mathrm{a}}$ & $11.49^{\mathrm{a}}$ & $5.05^{\mathrm{b}}$ \\
\hline & $3.57^{\mathrm{j}}$ & $4.821^{\mathrm{k}}$ & $8.068^{\mathrm{k}}, 6.987^{\mathrm{l}}$ & $6.78^{\mathrm{m}}$ & $8.77^{j}$ & $9.815^{\mathrm{k}}$ & $5.18^{\mathrm{m}}, 4.64^{\mathrm{e}}$ \\
\hline \multirow[t]{2}{*}{ Octahedral } & $3.964^{\mathrm{a}}$ & $6.060^{\mathrm{a}}$ & $7.095^{\mathrm{a}}$ & $6.723^{\mathrm{a}}$ & $9.067^{\mathrm{a}}$ & $11.68^{\mathrm{a}}$ & $5.21^{\mathrm{b}}$ \\
\hline & $3.62^{\mathrm{j}}$ & & $7.020^{1}$ & & $8.86^{\mathrm{j}}$ & & $5.34^{\mathrm{m}}, 4.94^{\mathrm{e}}$ \\
\hline
\end{tabular}

aDFT result (PLATO) from Ref. 31.

${ }^{b}$ Present work, VASP (PAW-GGA-PBE).

${ }^{c}$ Experimental data from Ref. 56.

${ }^{\mathrm{d}}$ Experimental data from Ref. 20.

eDFT (SIESTA) result from Refs. 28 and 29.

fPresent work, PLATO (GGA-PBE)

gModified embedded atom model calculation (Ref. 57).

hDFT (VASP) result from Ref. 60.

${ }^{\mathrm{i}} \mathrm{TB}$ result from Ref. 58.

jDFT (PWSCF) result from Ref. 30.

${ }^{\mathrm{k}}$ Finnis-Sinclair potential calculation (Ref. 22).

${ }^{1}$ DFT (VASP) result from Ref. 54.

mDFT (VASP) result from Ref. 59.

lybdenum, where Yamakawa et al. found $1.30 \pm 0.02 \mathrm{eV} .{ }^{64}$ The comparison of these two results suggests that the value of $1.28 \mathrm{eV}$ found in our calculations for the vacancy migration energy in Mo is in good agreement with the available experimental information.

Table I also gives the calculated formation and binding energies for divacancy defects involving the first-nearest- neighbor $(1 \mathrm{NN})$ and the second-nearest-neighbor $(2 \mathrm{NN})$ lattice sites for all the bcc transition metals. Again, we find a systematic group-specific trend for the divacancy energies that correlates well with the position of a bcc metal in the Periodic Table. Whereas the divacancy formation energies for the $2 \mathrm{NN}$ sites are lower than the corresponding values for the $1 \mathrm{NN}$ sites for all the elements of group 5B (vanadium, 


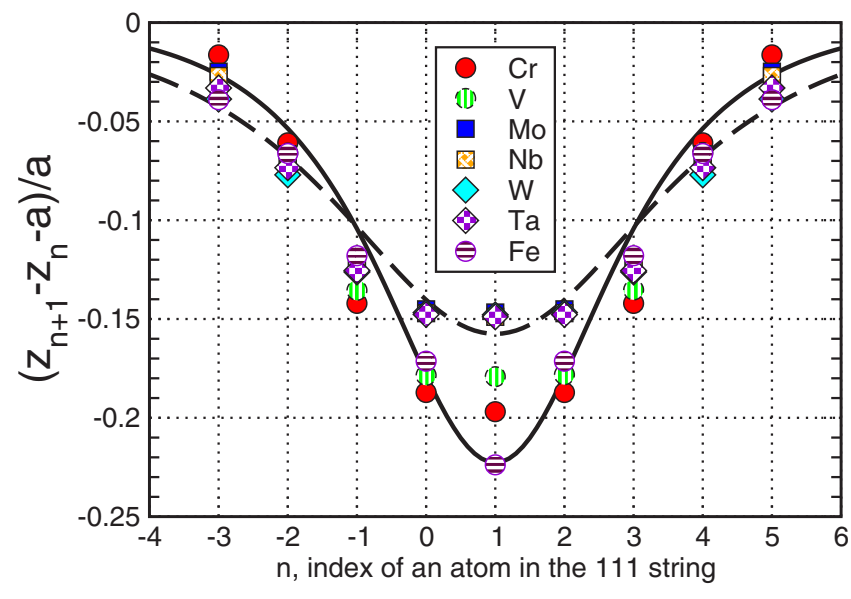

FIG. 2. (Color online) Atomic displacement fields in the central string of the $\langle 111\rangle$ crowdion self-interstitial atom configuration evaluated using density-functional theory for all the bcc transition metals. The solid and the dashed lines show the analytical soliton solutions of the sine-Gordon model [Eq. (33)] plotted for $\mathcal{N}=1.4$ and $\mathcal{N}=1.9$.

niobium, and tantalum), for all the elements of group 6B the $1 \mathrm{NN}$ divacancy formation energies are lower than the $2 \mathrm{NN}$ ones. For ferromagnetic bcc Fe, we find that the binding energy between the two $2 \mathrm{NN}$ vacancies is favored in comparison with those for the $1 \mathrm{NN}$ configuration, in agreement with earlier calculations performed using the SIESTA code. ${ }^{29}$ Our DFT results are at variance with predictions made by the semiempirical modified embedded atom method ${ }^{57}$ and by the tight-binding $(\mathrm{TB})^{58}$ potentials, where it was found that the $1 \mathrm{NN}$ divacancy formation energies are smaller than the formation energies of the $2 \mathrm{NN}$ divacancies for all the bcc transition metals. For the case of bcc Fe, by using a pairwise potential, Johnson ${ }^{65}$ found that the $2 \mathrm{NN}$ divacancy configuration was more stable than the $1 \mathrm{NN}$ divacancy configuration. We also note an experimental study of quenched highpurity tungsten carried out using a combination of field-ion microscopy (FIM) and electrical resistivity measurements. The study showed that the quenched-in defects observed by FIM were monovacancies and nearest-neighbor divacancies. ${ }^{66}$ The experiments give the value of $H_{v}^{f}$ $=3.6 \mathrm{eV}$ for the vacancy formation enthalpy and $H_{b}^{2 v}(1 N N)$ $=0.7 \mathrm{eV}$ for the the divacancy binding enthalpy. These values are in agreement with our DFT predictions for the vacancy and divacancy energies in bcc-W. We also note that the migration of divacancies in molybdenum is attributed to a resistivity recovery stage situated between 100 and $150{ }^{\circ} \mathrm{C}$ below stage III in electron irradiated molybdenum. ${ }^{67}$

Atomic displacements found in our DFT calculations for the most stable $\langle 111\rangle$ crowdion SIA configuration for the six bcc transition metals of groups $5 \mathrm{~B}$ and $6 \mathrm{~B}$ of the Periodic Table, as well the atomic displacements in a metastable $\langle 111\rangle$ crowdion in ferromagnetic Fe, are shown in Fig. 2. We find that the strongest $(\sim 20 \%)$ compressive deformation occurs in the core of a crowdion in the case of $3 d$ metals $(\mathrm{V}, \mathrm{Cr}$, and $\mathrm{Fe}$ ), while in the case of $4 d$ and $5 d$ metals the crowdions have a more extended core and the compressive strain in the core of the defect is lower $(\sim 15 \%)$. This finding suggests that the semicore electrons play a more significant part in determining the structure and the formation energy of SIA defects in the $4 d$ and $5 d$ transition metals in comparison with the $3 d$ metals.

The crowdion configuration can be described by the exactly solvable sine-Gordon model, ${ }^{31,35,68,69}$ which we also study in the last two sections of this paper. Figure 2 shows the field of atomic displacement calculated using the solution of the sine-Gordon model [see Eq. (33) below] plotted for two different values of the effective dimensionless width $\mathcal{N}$ of the crowdion configuration. We see that the extended core of the crowdion defect in bcc tungsten has a relatively large width $\mathcal{N}=1.9$, while in the case of bcc iron the solution of the sine-Gordon model provides the best fit to the DFT results if the width of the solution is taken as $\mathcal{N}=1.4$. Although the $\langle 111\rangle$ crowdion is not the most stable SIA defect configuration in bcc Fe, a cluster of crowdions is the same as a small dislocation loop similar to those observed by in situ transmission electron microscopy in iron. ${ }^{70}$ A cluster of crowdions is approximately described by solutions of the multistring Frenkel-Kontorova model. ${ }^{35}$

It is now well established that the group-specific dependence of properties of defects in bcc transition metals is correlated with their electronic structure and, specifically, with the number of valence $d$ electrons per atom. ${ }^{31,54,55,71} \mathrm{We}$ therefore performed a systematic study of the local density of states (LDOS) obtained from self-consistent DFT totalenergy calculations for the most stable SIA defect in bcc nonmagnetic transition metals, i.e., for the 111 crowdion configuration. Figure 3 shows the LDOS calculated for atoms forming the central $\langle 111\rangle$ string of crowdion configurations for all the bcc transition metals of groups $5 \mathrm{~B}$ and $6 \mathrm{~B}$. For a fully relaxed supercell with $128+1$ sites, there are five nonequivalent atoms in the central string containing the crowdion defect, starting with the atom in the center of the crowdion (in Fig. 3, it is denoted as atom 1) to the atom situated at the end of the string (denoted as atom 5). In each plot, the position of the Fermi energy is shown by a vertical solid line. A striking feature seen in Fig. 3 is the upward shift of the antibonding peak in the LDOS spectra of atoms situated near the center of crowdion configurations (atoms 1,2, and 3) in comparison with the bulk spectrum of DOS. The magnitude of this shift is $1.1,1.3,1.4,1.2,1.4$, and $1.7 \mathrm{eV}$ for vanadium, niobium, tantalum, chromium, molybdenum, and tungsten, respectively. The scale of this shift is sufficiently large, and it should be possible to observe it using the high-energy resolution electron energy loss spectroscopy technique. ${ }^{72,73}$ Our calculations show that the effect of the high strain associated with the core of an interstitial defect on the electronic structure is similar in terms of its magnitude to the chemical shift of electronic levels in the atoms forming the defect.

For an atom located sufficiently far away from the core of a crowdion (atom 5), the antibonding peak is shifted back to its bulk position. The position of the bonding peak of LDOS also varies depending on how far an atom is from the center of the defect configuration. Because of the strong compressive strain in the core of the crowdion defect, the effective local width of the $d$ band increases and the pseudogap practically vanishes for atoms 1,2 , and 3 that are near the center 

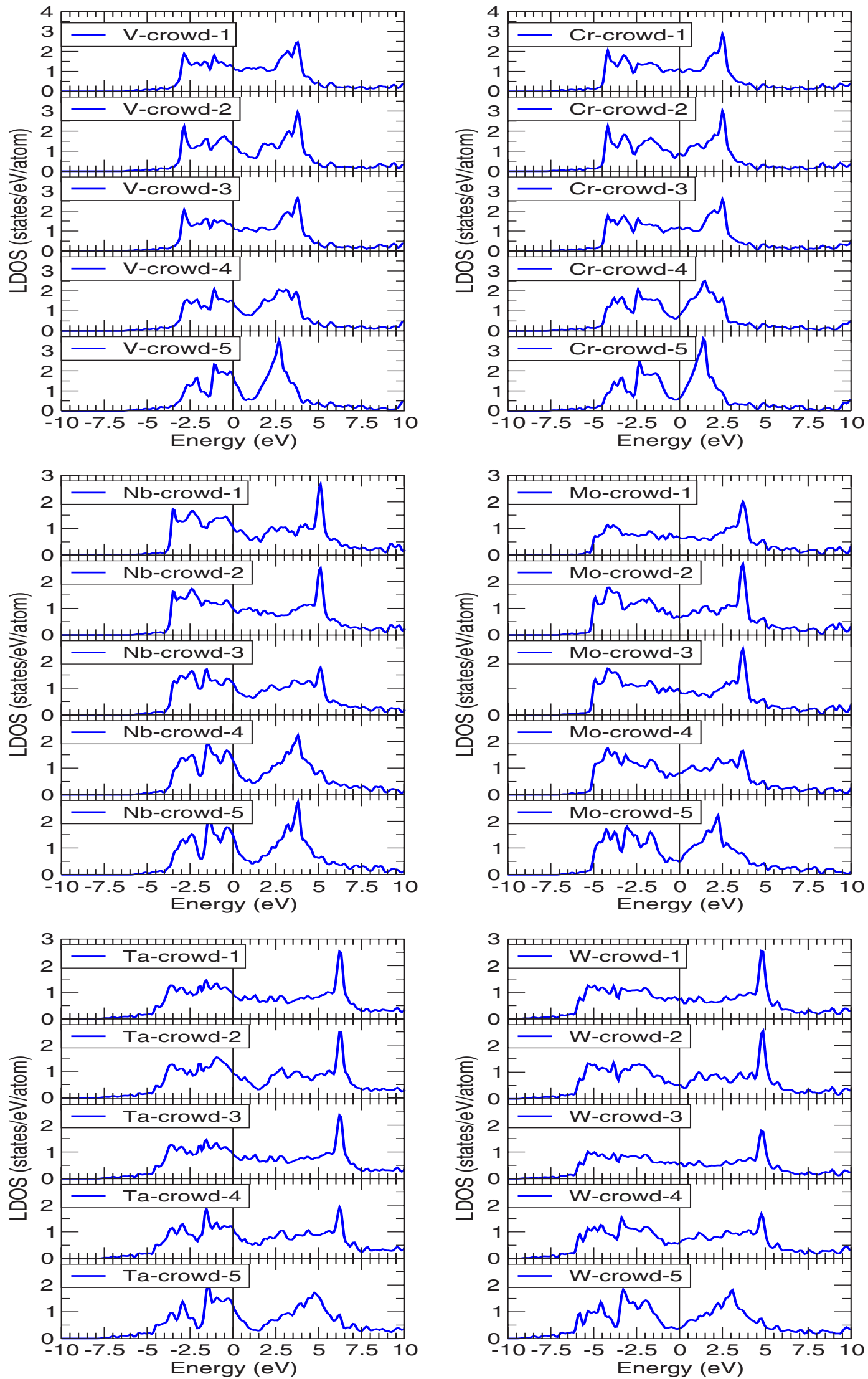

FIG. 3. (Color online) The local electronic density of states (LDOS) evaluated for the five atoms located in the central $\langle 111\rangle$ string of the crowdion configuration for each of the bcc metals forming groups V and IV of the Periodic Table. Calculations were performed using a $4 \times 4 \times 4128+1$ atom supercell. Atom 1 is situated in the center of the crowdion, and atom 5 is the furthest away from the center of the crowdion along the $\langle 111\rangle$ string. 
of the crowdion. The latter explains why the secondmoment-based interatomic potentials derived in the next section of the paper are able to provide a good fit to the formation energies of crowdions, as well as to other defect configurations investigated by DFT in the case of nonmagnetic bec transition metals.

Figure 4 shows LDOS plots for the three nonequivalent atoms belonging to one of the six nearest neighbor (NN) strings running parallel to the central string of the crowdion defect. Atom NN1 is located next to the center of the crowdion, whereas atoms NN2 and NN3 are situated further away along the same nearest-neighbor string. For comparison, at the top of the LDOS plots in Fig. 4 for each metal, we also show the corresponding bulk density of states. These bulk densities of states are dominated by the presence of the socalled pseudogap between the peaks of LDOS corresponding to bonding (at the bottom of the band) and antibonding (at the top of the band) states. Figure 4 shows that the shape of the LDOS for atoms in the NN strings is different from that of the LDOS for atoms forming the crowdion itself, and is quite similar to the bulk LDOS. Indeed, apart from a minor variation in the region just below the Fermi energy, we observe almost no change in the position of the antibonding peak in comparison with the bulk LDOS. This observation shows that the strong compressive deformations affecting the electronic structure of atoms in a crowdion defect are localized entirely within the central 111 strings and that the electronic structure of neighboring atomic strings is almost unaffected by the strain associated with the defect. This illustrates the short-range character of the effective interaction between atoms in bcc transition metals.

Figure 5 shows the LDOS calculated for both magnetic and nonmagnetic crowdion configurations in bcc-Fe. The positions of atoms in the latter are assumed to be the same as those found in a fully relaxed self-consistent spin-polarized calculation. LDOS plots for both the central and the NN strings of the crowdion are given in Fig. 5. This figure shows that $d$ orbitals give the dominant contribution to LDOS. The spin-polarized LDOS for the atom in the center of the defect shows why this atom has a negative magnetic moment $\left(-0.44 \mu_{B}\right)$ in comparison with the DFT value $\left(2.22 \mu_{B}\right)$ corresponding to bulk crystalline bcc-Fe. In the LDOS plot describing the central atom of the crowdion configuration, the Fermi energy is located to the left of the peak in the spin-up DOS, whereas the corresponding spin-down contribution has a pronounced bonding peak situated at the bottom of the band. As in the case of nonmagnetic bcc transition metals, the spin-polarized LDOSs for atoms forming the NN strings of the crowdion are almost unchanged in comparison with the bulk one. Due to the difference in the $d$-electron filling of Fe (group VIII in the Periodic Table of elements), the Fermi energy moves closer to the antibonding peak of the non-spinpolarized LDOS for atoms forming the central and the NN crowdion strings.

Figure 6 shows the LDOS evaluated for the 1NN and $2 \mathrm{NN}$ atoms surrounding a monovacancy for all the bcc transition metals of groups 5B and 6B. For each element, we also plot the total DOS averaged over all the 127 atoms in the supercell. We see that unlike the crowdion configuration, the LDOS plots for atoms in the vicinity of a vacancy defect show a strong similarity to the bulk DOS. At the same time, new features also emerge. For example, a new peak forms in the pseudogap of the DOS just below the Fermi energy for all the transition metals of group 6B. The same peak forms approximately $1 \mathrm{eV}$ above the Fermi energy in metals of group 5B.

LDOS plots for the $1 \mathrm{NN}$ and $2 \mathrm{NN}$ atoms show that it is their contribution to this new peak that differentiates the electronic contribution to the vacancy formation energy between the elements of groups 5B and 6B. Other peaks associated with the presence of the single vacancy defect can also be identified in Fig. 6 below the Fermi energy for all the bcc transition metals. The total vacancy DOS calculated here for bcc Ta and W are in agreement with earlier DFT (local density approximation) calculations performed using 53 atom supercells by Satta et al. $^{74}$

Finally, both spin-polarized and non spin-polarized LDOSs for the $1 \mathrm{NN}$ and $2 \mathrm{NN}$ atoms around the monovacancy in bcc-Fe are shown in Fig. 7. Again, the contribution of $d$ orbitals dominates the distribution of LDOS. We see a variation in the up spin contribution to the LDOS in the vicinity of $-2.0 \mathrm{eV}$ for both the $1 \mathrm{NN}$ and $2 \mathrm{NN}$ atoms. In the case of the non-spin-polarized calculation, this variation is seen in the pseudogap, which is now situated near $-1.0 \mathrm{eV}$.

\section{SEMIEMPIRICAL INTERATOMIC POTENTIALS}

The group 5B and 6B nonmagnetic bcc transition metals considered in the present work span three $d$-state electron filling factors with orbitals of varying spatial extents. From the previous section, changes in the fine structure of the local DOS at and around the $\langle 111\rangle$ interstitial defect show the presence of explicit $d$-state bonding effects that probably play some role in determining the absolute values of the corresponding defect energies. On the other hand, the universal ordering of the interstitial defect energies exhibited by all the nonmagnetic bcc metals considered here suggests that explicit angular-dependent interactions might not play a dominant role. Indeed, inspection of the local DOSs at and around an interstitial defect (see Fig. 3) reveals a general widening of the entire local DOS as one nears the core of the defect, suggesting that a simple scalar second-moment (or, equivalently, embedded atom model) representation might suffice to capture the relevant bonding mechanism responsible for the ordering of the defect energies. For these reasons, in the present work, we develop a parametrization of the FinnisSinclair-type empirical potential ${ }^{75,76}$ that models the band energy through a scalar second-moment representation. We take this simpler approach rather than develop, say, an empirical matrix potential, ${ }^{77}$ which does model an explicit $d$-state angular-dependent bonding.

Within the Finnis-Sinclair formalism, ${ }^{75}$ the total energy for an $\mathrm{N}$-atom system is given by

$$
E_{t o t}=\sum_{i}^{N} F\left(\rho_{i}\right)+\frac{1}{2} \sum_{i j, i \neq j}^{N} V\left(r_{i j}\right),
$$

where 

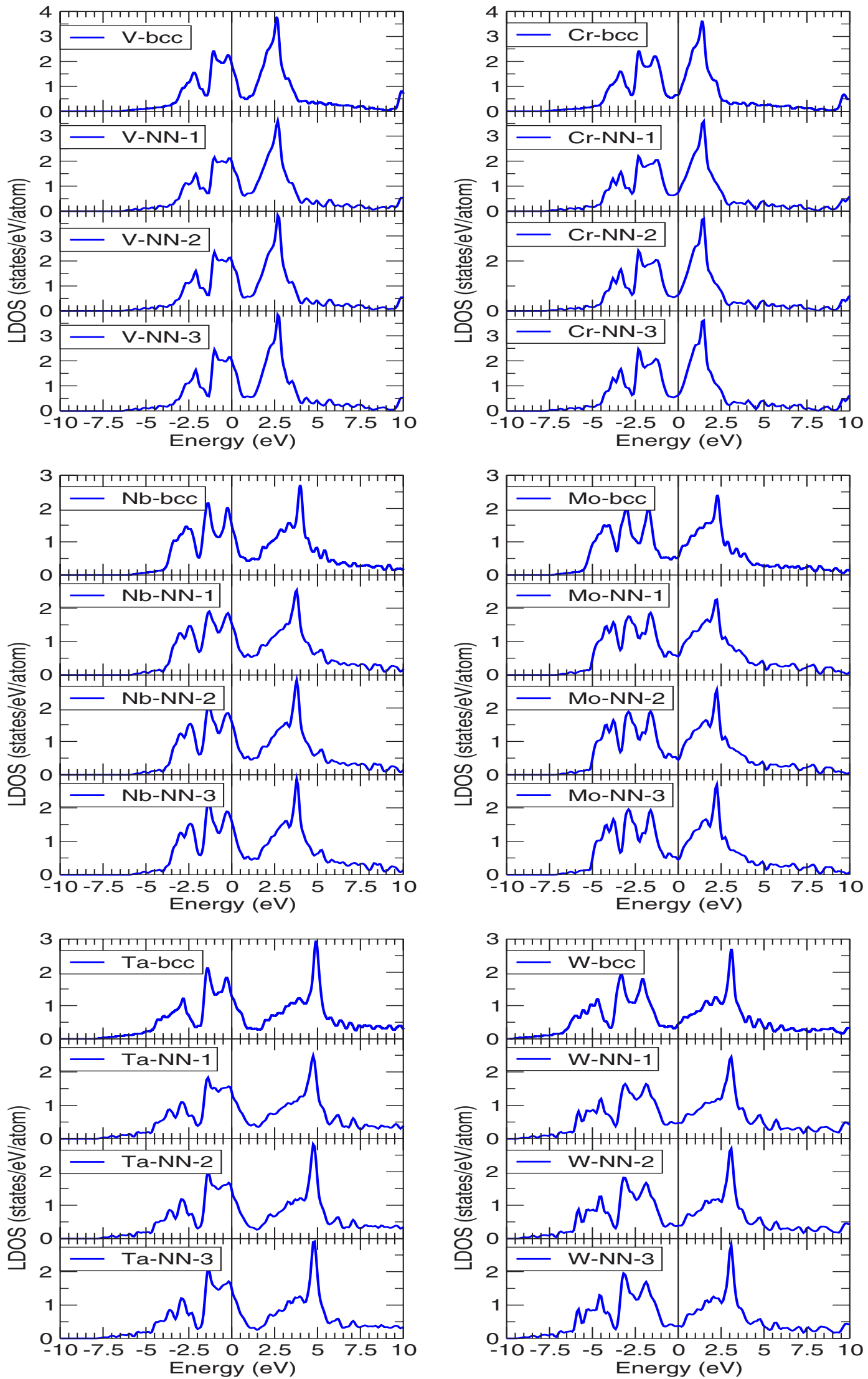

FIG. 4. (Color online) The local electronic density of states (LDOS) evaluated for the three atoms situated in one of the six nearestneighbor strings (adjacent to the central $\langle 111\rangle$ string) of the crowdion configuration for each of the bcc metals forming groups $\mathrm{V}$ and IV of the Periodic Table. Atom labeled NN-1 is the closest to the center of crowdion. 

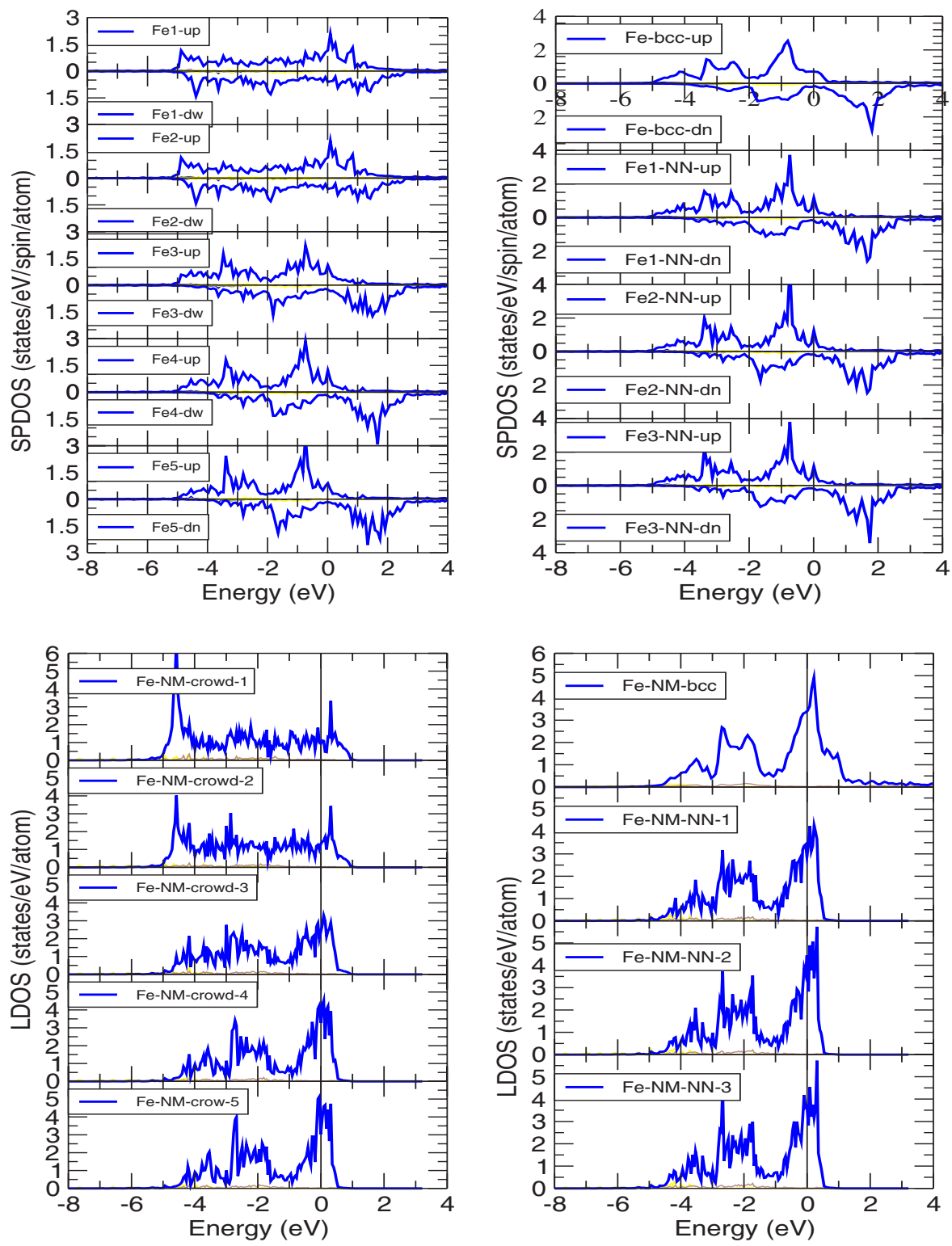

FIG. 5. (Color online) The local electronic density of states (LDOS) evaluated for the five atoms forming the central $\langle 111\rangle$ string of the crowdion defect in bcc iron, and the three atoms in one of the $\mathrm{NN}$ strings of the defect's configuration. Calculations were performed both for the spinpolarized and the nonmagnetic electronic configurations. The fixed atomic positions used in the nonmagnetic calculation are taken from the relaxed magnetic calculation. Contributions of $s, p$, and $d$ orbitals are shown, with $d$ orbitals giving the dominant contribution to the total LDOS.

$$
\rho_{i}=\sum_{j, j \neq i} f\left(r_{i j}\right)
$$

Here the (nonmagnetic) embedding energy $F(\rho)$ is given by

$$
F(\rho)=-A \sqrt{\rho}
$$

For this work, the pairwise density and the repulsive potential radial functions will be represented by a sum of cubic knot functions,

$$
f(r)=\sum_{n=1}^{N^{f}} f_{n}\left(r_{n}^{f}-r\right)^{3} \Theta\left(r_{n}^{f}-r\right)
$$

and where $\Theta(r)$ is a Heaviside step function defined as $\Theta(x)=1$ for $x>0$ and $\Theta(x)=0$ for $x<0$.

To obtain a useable empirical potential, the parameter $A$ and the knot coefficients $f_{n}$ and $V_{n}$ are optimized to reproduce as close as possible a variety of physical properties for the particular nonmagnetic bcc metal. For the present work, this physical data base contains the usual bulk equilibrium properties (cohesive energy, lattice constant, and elastic constants), the vacancy formation energy, and the interstitial defect energies listed in Table II. In addition to these bulk equilibrium properties, the potentials are also fitted to the metal's zero temperature isotropic thermal expansion coefficient defined via the thermodynamic relation 

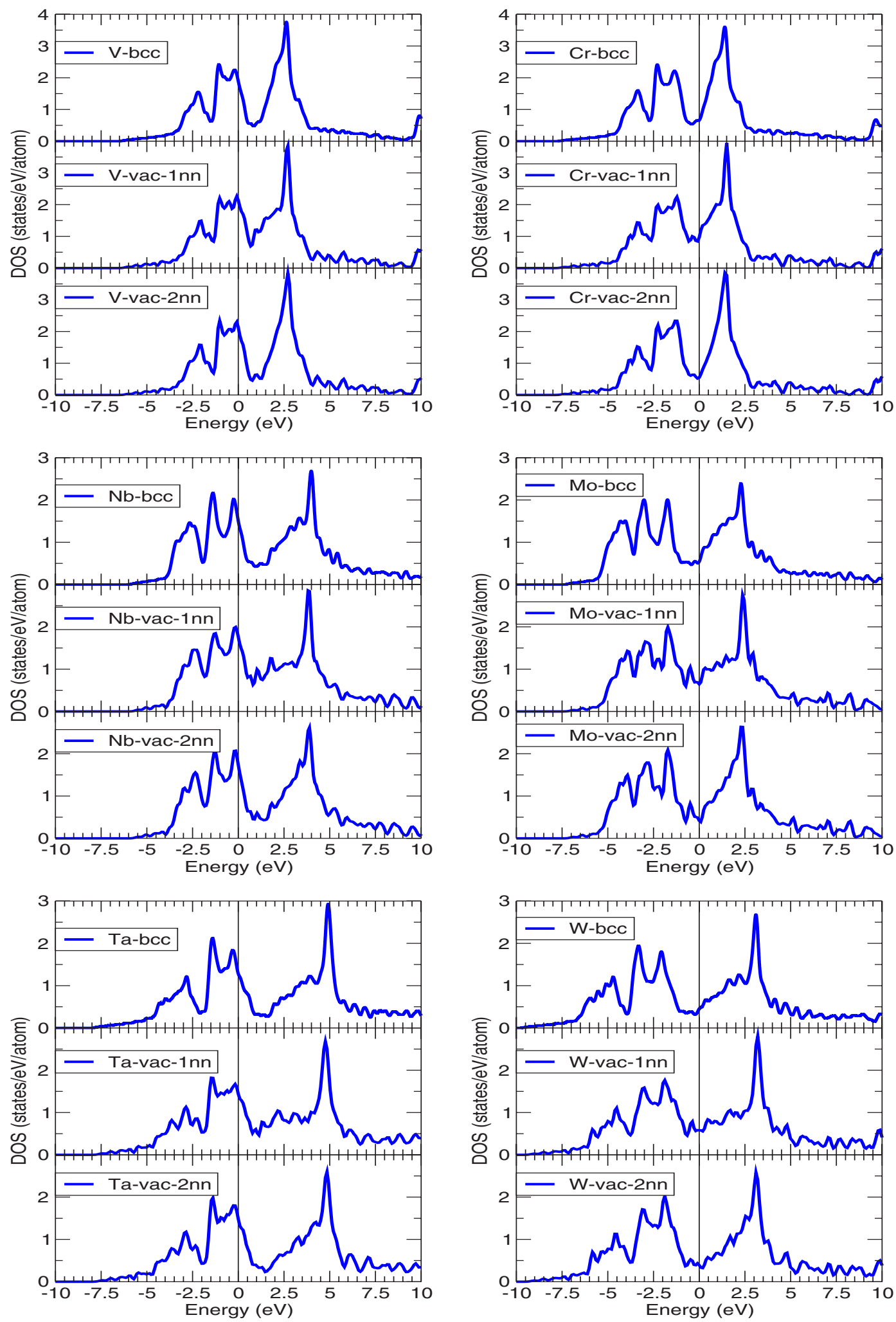

FIG. 6. (Color online) The local density of states for the $1 \mathrm{NN}$ and $2 \mathrm{NN}$ atoms of a vacancy evaluated for the six nonmagnetic bcc metals. Calculations were performed using a $4 \times 4 \times 4128-1$ atom supercell. The uppermost figure for each element shows the total density of states averaged over all the 127 atoms in the cell. 


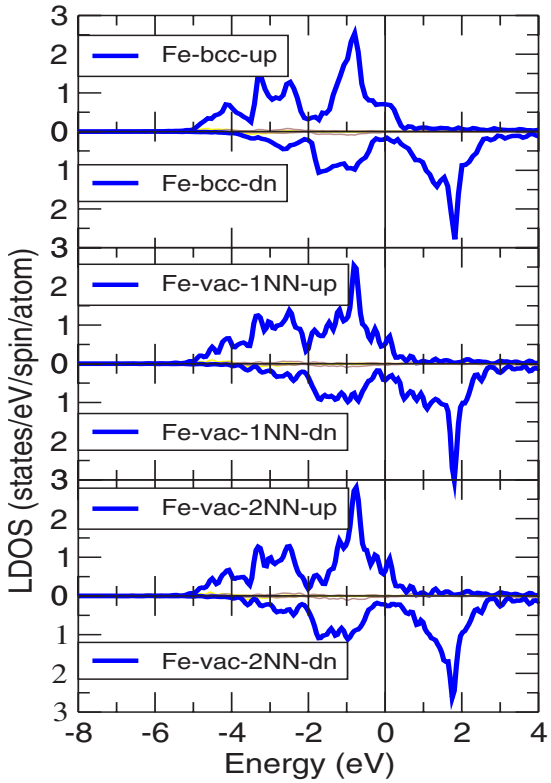

$$
\beta=\frac{1}{B}\left(\frac{\delta P}{\delta T}\right)_{V},
$$

where the thermodynamic pressure is determined from the constant temperature-volume derivative of the free energy

$$
P(T)=-\left(\frac{\delta F}{\delta V}\right)_{T} .
$$

Within the harmonic approximation, the classical free energy $F$ is given by

$$
F=E_{0}-k_{B} T \sum_{\lambda} \ln \left(\frac{\hbar \omega_{\lambda}}{k_{B} T}\right),
$$

where the summation is performed over the eigenmodes of the phonon spectrum of the material. Here, the first term
FIG. 7. (Color online) Same as in Fig. 6 but for bcc Fe. The figure shows results of both magnetic and nonmagnetic calculations of the LDOS for a monovacancy. The fixed atomic positions used in the nonmagnetic calculations are taken from the relaxed magnetic calculations.

TABLE II. Database values and the optimal values (in parentheses) evaluated using the empirical potentials for the nonmagnetic bcc metals considered in this work. The lattice constants $a_{0}$ are in angstrom, the elastic constants are in $\mathrm{GPa}$, and all the energies are given in $\mathrm{eV}$. The bcc bulk equilibrium properties are taken from Ref. 75 and the defect energies from Table I.

\begin{tabular}{llllll}
\hline \hline & \multicolumn{1}{c}{$\mathrm{V}$} & \multicolumn{1}{c}{$\mathrm{Nb}$} & \multicolumn{1}{c}{$\mathrm{Ta}$} & \multicolumn{1}{c}{$\mathrm{Mo}$} & \multicolumn{1}{c}{$\mathrm{W}$} \\
\hline$a_{0}$ & 3.0399 & 3.3008 & 3.3058 & 3.1472 & 3.1652 \\
$E_{c}$ & -5.31 & -7.57 & -8.1 & -6.82 & -8.9 \\
$B$ & 155.1 & 171.0 & 196.1 & 262.6 & 310.4 \\
$C^{\prime}$ & 42.6 & 28.1 & 82.4 & 108.9 & 160.6 \\
$C_{44}$ & 54.6 & 56.7 & 52.6 & 151.6 & 159.0 \\
$\beta\left(10^{-4}\right)$ & $0.252(1.28)$ & $0.219(0.57)$ & $0.189(0.107)$ & $0.144(0.154)$ & $0.135(0.120)$ \\
$E_{\text {vac }}$ & $2.51(2.51)$ & $2.99(2.99)$ & $3.14(3.14)$ & $2.96(2.96)$ & $3.56(3.56)$ \\
$E_{d 111}$ & $3.367(3.375)$ & $5.253(5.203)$ & $5.832(5.775)$ & $7.417(7.406)$ & $9.548(9.550)$ \\
$E_{d 110}$ & $3.652(3.642)$ & $5.597(5.684)$ & $6.382(6.414)$ & $7.581(7.581)$ & $9.844(9.841)$ \\
$E_{d 100}$ & $3.918(3.921)$ & $5.949(6.005)$ & $7.003(6.955)$ & $9.004(9.004)$ & $11.490(11.513)$ \\
$E_{\text {crd }}$ & $3.371(3.379)$ & $5.254(5.255)$ & $5.836(5.873)$ & $7.419(7.431)$ & $9.551(9.557)$ \\
$E_{\text {tet }}$ & $3.835(3.819)$ & $5.758(5.733)$ & $6.771(6.631)$ & $8.401(8.400)$ & $11.050(10.999)$ \\
$E_{\text {oct }}$ & $3.964(3.967)$ & $6.060(6.009)$ & $7.095(7.134)$ & $9.067(9.067)$ & $11.680(11.709)$ \\
\hline \hline
\end{tabular}


solely from the volume dependence of the phonon frequencies,

$$
P=-k_{B} T \sum_{i} \frac{1}{\omega_{i}} \frac{\delta \omega_{i}}{\delta V} .
$$

From Eq. (6), the thermal expansion coefficient thus reduces to

$$
\beta=\frac{k_{B}}{B V} \sum_{i} \gamma_{i}
$$

where the $\gamma_{i}=-\left(V / \omega_{i}\right)\left(\delta \omega_{i} / \delta V\right)$ are the so-called Grüneisen parameters. ${ }^{80}$

Table II lists the physical data base values for the transition metals as well as the corresponding values obtained for the optimal fits. For the knot points, a set of equally spaced points was chosen, with the outermost point being located at an approximately halfway distance between the second- and third-nearest-neighbor shells for the repulsive potential, and just beyond the second-nearest-neighbor shell for the pairwise density function. This was first done for vanadium and then scaled for the other elements according to their equilibrium lattice constants. The bulk equilibrium bcc lattice constant, cohesive energy, and elastic constants were exactly reproduced using a linear matrix solution to determine the values of $A$ and the outermost four knot coefficients of the repulsive potential. The remaining knot coefficients of the the repulsive potential and those of the pairwise density term were determined via the numerical minimization of a weighted objective function, where for each candidate parametrization the vacancy and the interstitial defect structures were relaxed to determine their formation energies. A periodic $5 \times 5 \times 5$ unit cell was used to represent these defect structures. For the final interstitial defect optimization, a number of short-range knot functions were added to obtain the required accuracy. In addition to the above physical properties, the potentials were also optimized so that the bcc structure was more stable against the fcc structure by at least $0.1 \mathrm{eV}$. The numerical minimization was performed via a combination of simulated annealing ${ }^{81}$ and a nonlinear downhill Simplex algorithm, ${ }^{82}$ both of which do not employ derivative information. The weights for each physical property were varied throughout the fitting procedure according to emergent correlations between fitting variables and database values, with the final weighting biasing the interstitial energies, followed by the vacancy energy, the zero Kelvin thermal expansion coefficient, and the fcc-bcc equilibrium cohesive energy difference. The optimal parameter sets for all the metals can be found in the Appendix. No satisfactory fit could be found for $\mathrm{Cr}$ that described well both its bulk equilibrium properties and its defect energies.

The inclusion of the thermal expansion coefficient within the physical database results in higher than second order derivative information now being included within the fitting procedure. This is arguably an essential requirement for the development of empirical potentials based on a range of accurate DFT defect data since such an approach begins to include anharmonic information-phonon-phonon interactions-an increasingly important aspect when thermal
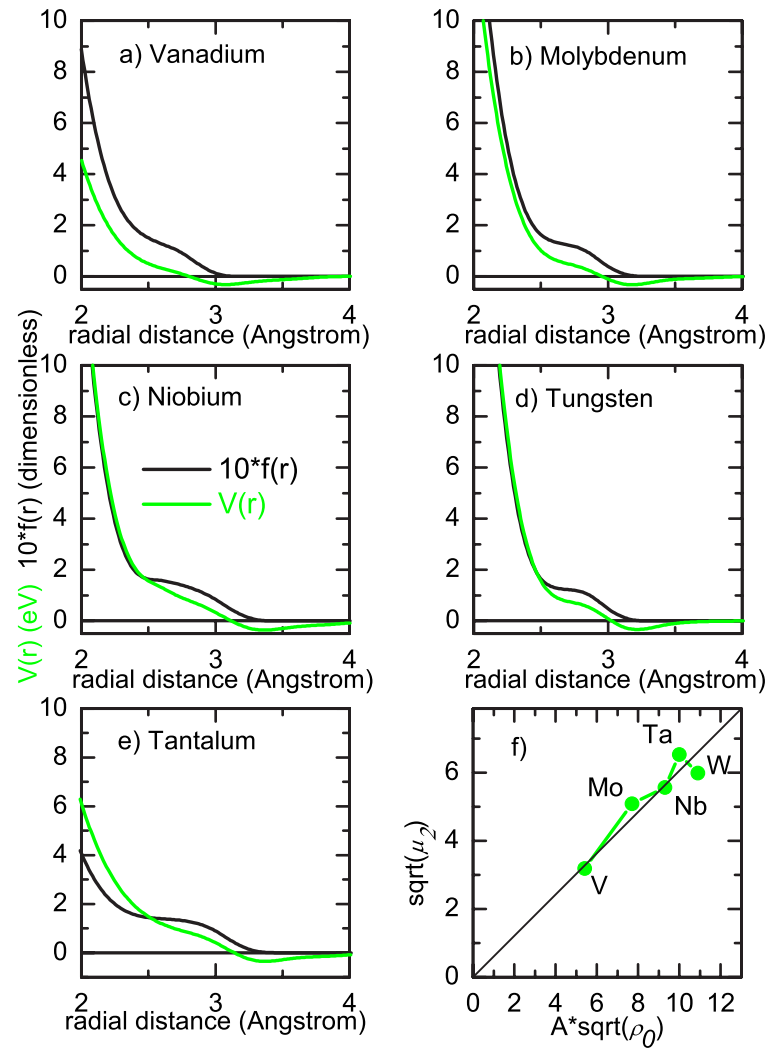

FIG. 8. (Color online) [(a)-(e)] Plot of pairwise density and repulsive potential radial functions of optimized fits. Note that the pairwise density is multiplied by a factor of 10. (f) Plots the DFTderived second moments of the density of states as a function of the product of the empirical potential parameter $A$ and the square root of the effective density $\rho_{0}(=1)$ corresponding to the equilibrium lattice constant for bcc crystal lattice.

entropy effects of strongly distorted defect structures are to be modeled. These considerations, along with the dynamical weight adjustments during the fitting procedure, constitute important new developments in the construction of modern empirical potentials.

Figures $8(a)-8(e)$ display the pairwise density and the repulsive potential radial functions for all the five metals considered here. We see that the corresponding functions are all qualitatively similar, where the pairwise density function monotonically increases as a function of decreasing distance and exhibits a plateau region between the first and the second nearest-neighbor shells. In the case of the repulsive potential, all the metals exhibit an outermost attractive part. These qualitative features are also seen in a magnetic potential for bcc Fe, ${ }^{83}$ which used a similar fitting procedure with a different embedding function that also included a magnetic energy contribution. As one moves, within a group, down the periodic table, both radial functions appear harder at a shorter range. The exception to this trend is tantalum, whose optimal fit always converged to a "softer" shorter range functional dependence. In the present work, we consider only interstitial defect migration and, therefore, presently do not include a short-range correction to the potentials, which is necessary in studying high-energy collision dynamics. Thus, 

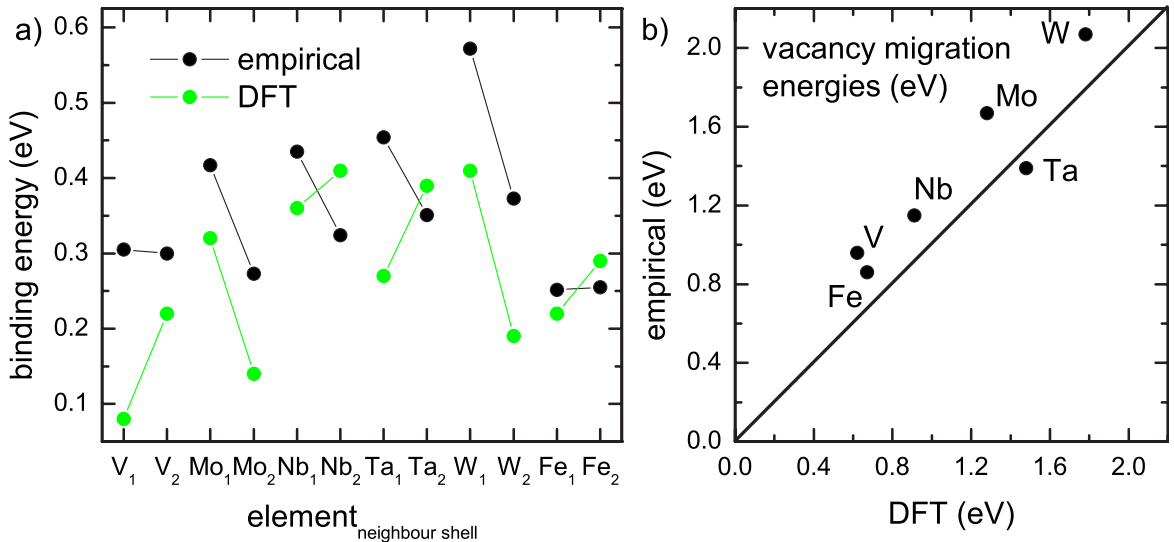

FIG. 9. (Color online) (a) Plot of predicted first nearest and second nearest divacancy binding energies and (b) vacancy migration energies using the current empirical potentials and DFT data obtained from Table I. the potentials presented here are currently not suitable for simulating high-energy collision cascades.

Figure 8(f) displays the square root of the second moment of the DFT derived $d$-band density of states (shown in Fig. 3) plotted for each metal as a function of the empirical fitting parameter $A$ multiplied by the square root of the equilibrium density $\rho_{0}$, where the latter has been chosen to equal unity for all elements. We remind the reader that within the FinnisSinclair approximation, the latter quantity represents the electronic band contribution to the cohesive energy and is assumed to be proportional to the square root of the second moment of the DOS. In this context, the quantity $A \sqrt{\rho_{0}}$ depends partly on the number of $d$ electrons and partly on the shape of the density of states for each corresponding element. That a relatively good linear relationship is seen in Fig. 8(f) indicates that the empirical fits are, to a certain degree, systematic across the series of metals considered in this study. This result also demonstrates that the much larger bandwidth associated with the long range $s$ electrons can be well absorbed into the repulsive pair potential term, resulting in the longer range attractive tail seen for all elements in Figs. 8(a)-8(e).

Figure 9(a) displays the predicted first- and secondnearest-neighbor divacancy binding energies using the presently developed empirical potentials. For comparison, the DFT calculated values from Table I are also shown. We also include similar data for bcc-Fe using the magnetic potential. ${ }^{83}$ For the empirical potential calculations, a 10 $\times 10 \times 10$ supercell was used. Inspection of this data reveals that for all empirical potentials, the binding energy is positive and is in reasonable agreement with DFT. In particular, the group 6B elements reproduce the first- and secondnearest-neighbor ordering, as predicted by DFT. Figure 9(b) now plots the predicted monovacancy migration energy using the nudged elastic band method calculated by the empirical potentials ${ }^{84}$ as a function of the corresponding DFT values from Table I. We see a good systematic agreement with all elements, where the empirical potential values slightly overestimate the DFT values. The exception is tantalum, which slightly underestimates the corresponding DFT value. When comparing the predictions of such empirical potential models with DFT, it must be remembered that the former constitutes a significant simplification of the bonding physics between atoms, and thus the accuracy of such models cannot and should not be expected to be more than approximately $0.1 \mathrm{eV}$.

\section{DYNAMICS OF DEFECTS AT ELEVATED TEMPERATURES}

In the previous sections, we described how densityfunctional calculations provided a foundation for constructing a family of semiempirical many-body potentials for modeling equilibrium properties and defect configurations in several nonmagnetic bcc metals, including tungsten. We now investigate the dynamics of single interstitial crowdion defects at elevated temperatures, using tungsten as a case study. We choose tungsten because of its relevance to applications in fusion as a plasma-facing material and also because of its position at the bottom of group 6B of the Periodic Table. Since an extensive study of the dynamical behavior of defects in vanadium was already performed in Ref. 38, by investigating the dynamics of defects in tungsten, we hope to be able to identify common generic features characterizing the thermally activated migration of defects in the two bcc metals. Since vanadium and tungsten are situated at the opposite ends of the "spectrum" of nonmagnetic bcc metals, similarities between the types of dynamical behavior of defects in vanadium and tungsten found in simulations will likely apply to all the other nonmagnetic bcc metals.

Figures 10-13 show examples of trajectories of migration of a crowdion defect in tungsten simulated using molecular dynamics. The position of the center of a crowdion was followed over intervals of time of the order of $1 \mathrm{~ns}$ at a constant temperature using a thermally equilibrated simulation cell containing approximately 60000 atoms.

In the case of a very low temperature $(T=100 \mathrm{~K})$, illustrated in Fig. 10, the defect spends most of its time in one of the shallow local minima of the Peierls potential and performs occasional hops between the adjacent minima in this potential. The Peierls potential models the effect of discreteness of the lattice on the translational motion of defects in the [111] direction. The fact that the curves describing the Cartesian coordinates of the center of the defect, $X(t), Y(t)$, and $Z(t)$, in Fig. 10 are identical confirms that the migration of the crowdion at this temperature only involves translation of the defect along its axis in the [111] direction.

At a higher temperature $T=400 \mathrm{~K}$, the pattern of migration shown in Fig. 11 becomes more random and the presence of the Peierls potential is no longer significant. The migration of a crowdion at $T=400 \mathrm{~K}$ remains one dimensional, and the defect performs stochastic longitudinal 


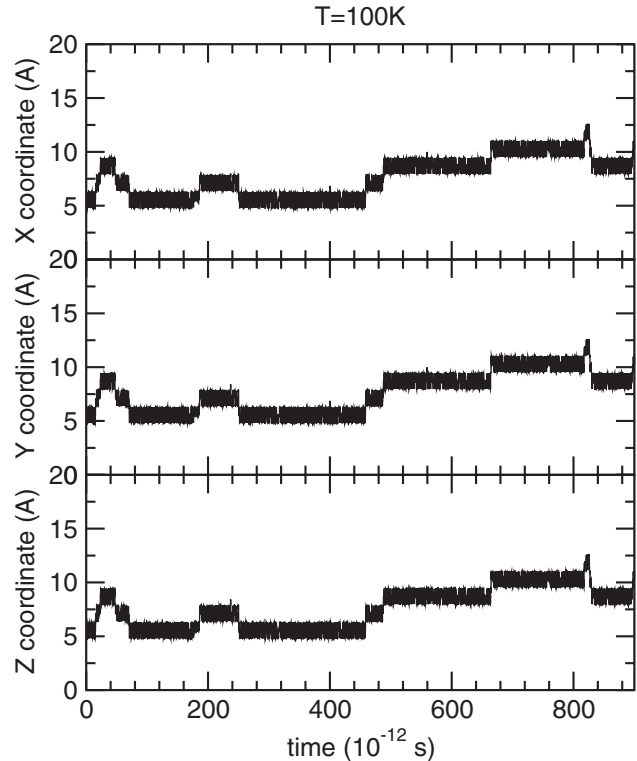

FIG. 10. The trajectory of the center of a $\langle 111\rangle$ crowdion in tungsten at $T=100 \mathrm{~K}$ simulated using the semiempirical many-body potential described in the Appendix.

Brownian motion in the direction of its axis. At an even higher temperature $T=800 \mathrm{~K}$ (see Fig. 12), we observe the infrequent occurrence of a type of dynamical event, namely, the transformation of the [111] crowdion into one of the three alternative crystallographically equivalent configurations:

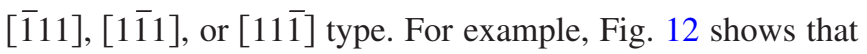
a crowdion that initially moves in the [111] direction then undergoes a transition into a $[\overline{1} 11]$ configuration at $t$ $\approx 320$ ps.

In the limit of very high temperatures illustrated in Fig. 13, the Brownian motion of the defect becomes effectively three dimensional, with the axis of the crowdion changing its orientation approximately once every $10 \mathrm{ps}$.

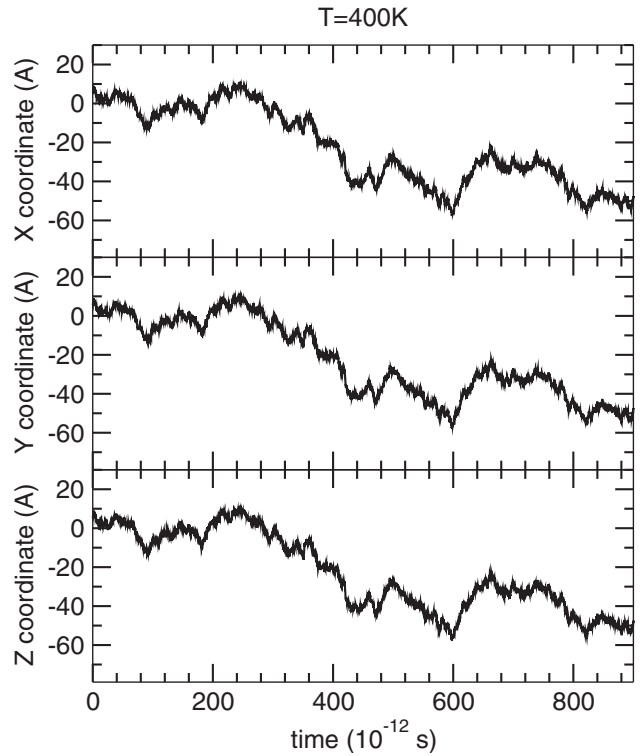

FIG. 11. Same as in Fig. 10 but for $T=400 \mathrm{~K}$.

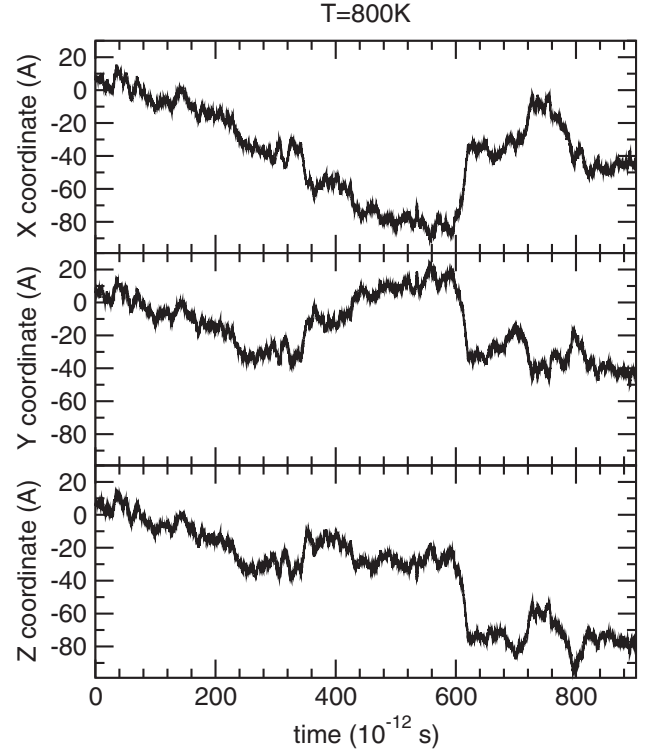

FIG. 12. Same as in Fig. 10 but for $T=800 \mathrm{~K}$.

Figure 14 shows the frequency of events of the virtual formation of the $\langle 110\rangle$-type configurations during the migration of a crowdion in tungsten at elevated temperatures. Since a $\langle 110\rangle$-type configuration is an intermediate configuration for the event of rotation of the axis of a crowdion between two crystallographically equivalent [111] directions, the frequency of occurrence of the $\langle 110\rangle$ configurations is a convenient measure of the frequency of rotations of the axis of a migrating crowdion. We observed no virtual $\langle 110\rangle$ configuration on the time scale of molecular dynamics simulations performed in this work for temperatures below $500 \mathrm{~K}$. However, by extrapolating the high-temperature data we find that even at room temperature, a crowdion defect changes the orientation of its axis approximately $1.35 \times 10^{6} / \mathrm{s}$. Observing this in a molecular dynamics simulation at $T=300 \mathrm{~K}$ would require extending the simulation time to several hundred

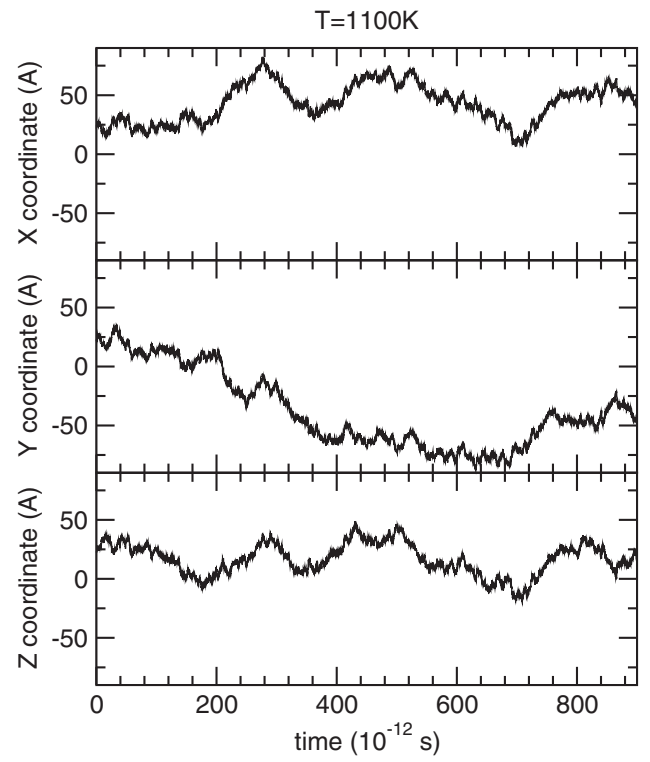

FIG. 13. Same as in Fig. 10 but for $T=1100 \mathrm{~K}$. 


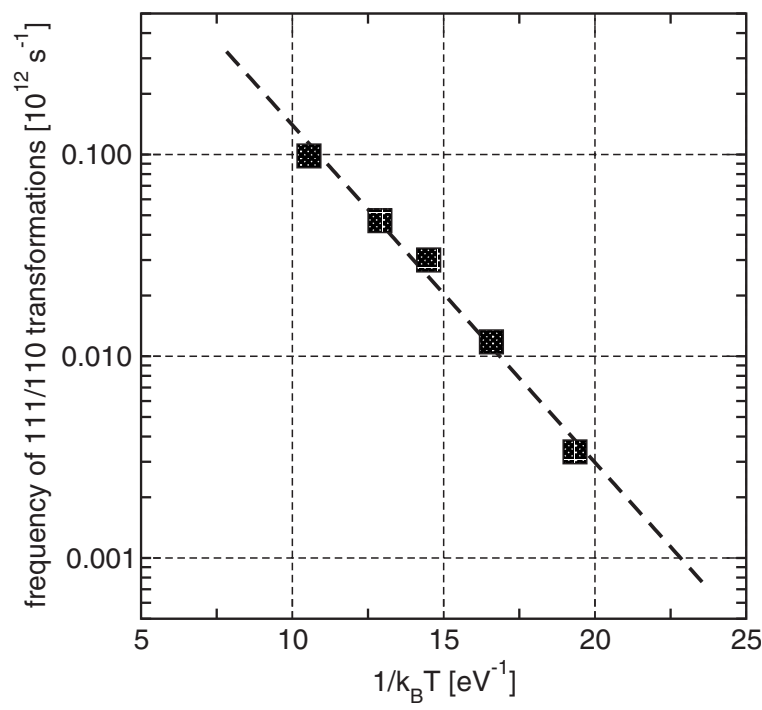

FIG. 14. The frequency of the $\langle 111\rangle \leftrightarrow\langle 110\rangle$ transformation observed in molecular dynamics simulations and plotted as a function of absolute temperature $T$. The dashed line corresponds to $\nu_{r}(T)$ $=6.59 \times 10^{12} \exp \left(-[0.385 \mathrm{eV}] / k_{B} T\right) / \mathrm{s}$. The Debye temperature $T_{D}$ of tungsten is $T_{D}=310 \mathrm{~K}$. This corresponds to $1 / k_{B} T_{D}$ $=37.43 \mathrm{eV}^{-1}$.

nanoseconds (i.e., by more than 2 orders of magnitude).

The effective activation energy $\Delta E_{r}$ characterizing the temperature dependence of the frequency of rotations found in simulations is $\Delta E_{r} \approx 0.38 \mathrm{eV}$. The $0.4 \mathrm{eV}$ activation energy for rotations, which is very similar to the energy found in molecular dynamics, was also found using the nudged elastic band method for a pathway linking two crystallographically equivalent 'rotated' crowdion configurations.

The prefactor $\nu_{0}$ in the Arrhenius formula for the frequency of rotations of the axis of the crowdion

$$
\nu_{r}(T)=\nu_{0} \exp \left(-\frac{\Delta E_{r}}{k_{B} T}\right)=6.59 \times 10^{12} \exp \left(-\frac{0.385 \mathrm{eV}}{k_{B} T}\right)
$$

is approximately equal to 0.16 of the Debye frequency of tungsten $\omega_{D}=4.05 \times 10^{13} / \mathrm{s}$. Similarly, the prefactor $\nu_{0}$ in the expression for the frequency of rotations of crowdions in vanadium $\nu_{0}=1.3 \times 10^{13} / \mathrm{s}$ found in Ref. 38 also approximately equals one-quarter of the Debye frequency of vanadium $\omega_{D}=5.1 \times 10^{13} / \mathrm{s}$. Since the Debye frequency equals $2 \pi$ divided by the period $\tau$ of vibrations of atoms in the lattice, the attempt frequency $\nu_{0}=1 / \tau$ in the limit of high temperatures can be estimated as $\nu_{0}=\omega_{D} / 2 \pi \approx 0.16 \omega_{D}$. This estimate agrees with the attempt frequency factor found in our simulations.

To investigate the statistical properties of the Brownian motion of crowdions, we evaluated the coefficient of diffusion of crowdions using trajectories of defects similar to the ones shown in Figs. 10-13. Following Guinan et al., ${ }^{85}$ for each temperature, we used a single long trajectory $\mathbf{R}(t)$ and divided it into smaller time intervals, which were treated as statistically independent realizations of the stochastic Brownian motion. To improve the convergence of the algorithm, we ensured that the average position of the particle on any chosen time interval satisfied the condition $\overline{\mathbf{R}(t)}=0$. This was achieved by subtracting the local "drift" term $\mathbf{R}_{\text {drift }}(t)$ $=\mathbf{R}\left(t_{0}\right)+\mathbf{V}_{\text {drift }}\left(t-t_{0}\right)$ from the actual time-dependent position of the defect $\mathbf{R}(t)$. The magnitude of the drift velocity was determined by the condition that the stochastic process $\mathbf{R}(t)-\mathbf{R}_{\text {drift }}(t)$ had zero mean. By following this approach, we ensured that only local fluctuations of the position of the defect contributed to the coefficient of diffusion. In the limit $t \rightarrow \infty$, our treatment satisfies the condition that is implicit in any treatment of Brownian motion, namely, that on average the Brownian particle remains at the origin $\overline{\mathbf{R}(t)}=0$, see, for example, Refs. 86 and 87.

To show the mathematical equivalence between our method and the conventional approach to evaluating the diffusion coefficient, we consider the definition of $D$ (see, e.g., Refs. 85 and 88),

$$
D=\lim _{t \rightarrow \infty} \frac{\overline{\mathbf{R}^{2}(t)}}{6 t}
$$

where the bar denotes taking the statistical average over an ensemble of realizations of the stochastic trajectory of the defect $\mathbf{R}(t)$. The definition (11) is subject to a condition that the Brownian motion is random, i.e., that for any $t>0$

$$
\overline{\mathbf{R}(t)}=0 \text {. }
$$

The latter condition implies that, on average, a Brownian particle does not drift away from its initial position $\mathbf{R}(t=0)$, which we take as the origin of the Cartesian system of coordinates. In a typical molecular dynamics simulation, the above condition (12) is not satisfied, and, on average (even if taking the average involves subdividing a trajectory of the defect into independent segments), the defect drifts away from its initial position. The evaluation of the diffusion coefficient involves taking the square of the coordinate of the defect [Eq. (11)] and the occurrence of this drift contributes to the statistical error in the calculated value of $D$. How do we eliminate the effect of this systematic drift? Combining the ergodic hypothesis and Eq. (12), we equate the ensemble and the time average coordinates of the defect,

$$
\overline{\mathbf{R}}=\lim _{t \rightarrow \infty} \frac{1}{t} \int_{0}^{t} \mathbf{R}\left(t^{\prime}\right) d t^{\prime}=0
$$

This condition means that in the limit $t \rightarrow \infty$,

$$
\int_{0}^{t} \mathbf{R}\left(t^{\prime}\right) d t^{\prime}=\mathbf{o}(t)
$$

Now, if instead of a real trajectory $\mathbf{R}(t)$ of a defect found in a molecular dynamics simulation, we construct a function

$$
\mathbf{R}(t)-\frac{2 t}{\tau^{2}} \int_{0}^{\tau} \mathbf{R}\left(t^{\prime}\right) d t^{\prime}
$$

which has zero mean on the interval $0<t<\tau$, and substitute it into definition (11), we arrive at 


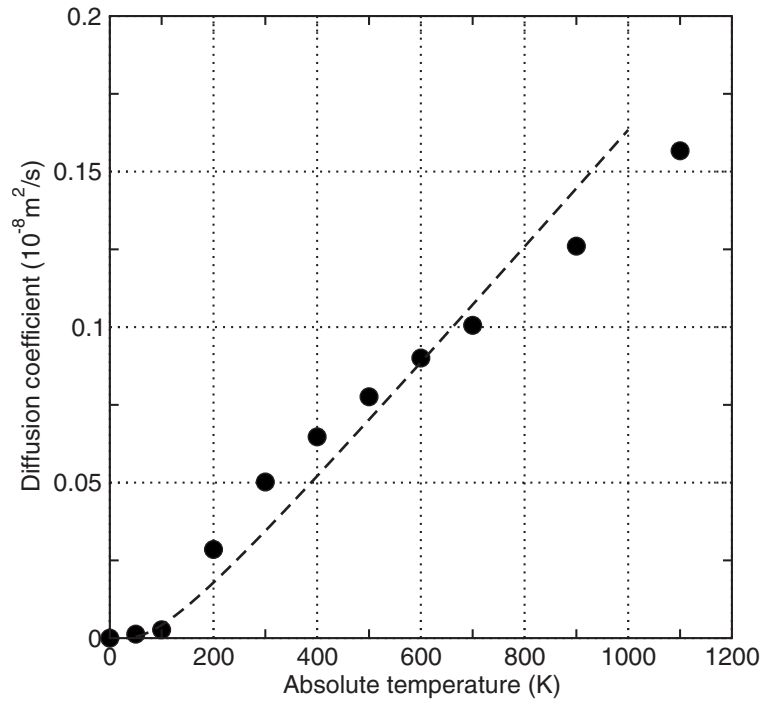

FIG. 15. Coefficient of diffusion of crowdions found using molecular dynamics simulations and plotted as a function of the absolute temperature $T$. The dashed line is given by an analytical formula $D(T)=\left(1.9 \times 10^{-4}\right) T \exp \left(-[0.013 \mathrm{eV}] / k_{B} T\right) \mathrm{m}^{2} / \mathrm{s}$. The Debye temperature $T_{D}$ of tungsten is $T_{D}=310 \mathrm{~K}$.

$$
\begin{aligned}
\lim _{t \rightarrow \infty} \frac{\overline{\left[\mathbf{R}(t)-\frac{2 t}{\tau^{2}} \int_{0}^{\tau} \mathbf{R}\left(t^{\prime}\right) d t^{\prime}\right]^{2}}}{6 t} \\
\quad=\lim _{t \rightarrow \infty} \frac{\overline{\left[\mathbf{R}(t)-\frac{2}{t} \int_{0}^{t} \mathbf{R}\left(t^{\prime}\right) d t^{\prime}\right]^{2}}}{6 t}=\lim _{t \rightarrow \infty} \frac{\overline{\left[\mathbf{R}(t)-\frac{\mathbf{o}(t)}{t}\right]^{2}}}{6 t} \\
=D .
\end{aligned}
$$

In other words, by subtracting the local drift motion from realizations of Brownian trajectories of a defect on finite intervals of time, we do not alter the calculated value of the diffusion coefficient in comparison with the original formula (11). Numerical simulations show that the use of Eq. (15) reduces the statistical uncertainty of the calculated value of $D$ and improves the numerical convergence of the method.

The coefficient of diffusion of crowdions determined from trajectories of defects simulated for several temperatures in the range $50 \mathrm{~K}<T<1100 \mathrm{~K}$ is shown in Fig. 15. The diffusion coefficient exhibits three different types of behavior as a function of absolute temperature. In the low-temperature region $T \leqslant 200 \mathrm{~K}$, diffusion is thermally activated. The activation energy is fairly small $E_{a} \sim 0.013 \mathrm{eV}$. In the region $200 \mathrm{~K} \leqslant T \leqslant 600 \mathrm{~K}$, crowdions migrate one dimensionally, and the character of diffusion resembles that of a free particle moving in a viscous medium under the influence of a random thermal force. For temperatures higher than approximately $600 \mathrm{~K}$, we start seeing the effect of rotations of the axis of the migrating crowdion defect. This involves the formation of virtual saddle-point $\langle 110\rangle$-like configurations that effectively slow the diffusion down. For temperatures higher than $600 \mathrm{~K}$, diffusion of crowdions is no longer one dimensional, and the change of the slope of function $D(T)$ at $T \sim 600 \mathrm{~K}$ indicates the transition to the more three-dimensional diffusion of defects that involves intervals of rapid onedimensional migration in the $\langle 111\rangle$ directions and occasional reorientations of the axis of crowdions occurring with a frequency that increases as a function of temperature. Soneda and Diaz de la Rubia ${ }^{89,90}$ found a similar pattern of diffusion for the case of clusters of interstitial defects in iron, but not for single interstitial atom defects.

The coefficient of diffusion as a function of temperature shown in Fig. 15 is entirely different from that found by Guinan et al. ${ }^{85}$ for tungsten (see, e.g., Fig. 4 of Ref. 85). The origin of the difference is traced to the fact that the semiempirical potential used by Guinan et al. predicted the $\langle 110\rangle$ rather than the (correct) $\langle 111\rangle$ configurations as the lowestenergy structure of a self-interstitial atom defect. Since the migration of a $\langle 110\rangle$ dumbbell is characterized by a high activation energy barrier $E_{a} \sim 0.365 \mathrm{eV}$, the diffusion coefficient found in Ref. 85 is many orders of magnitude smaller than that found in our simulations. Similarly, in simulations of interstitial defects in tungsten performed by Carlberg et $a l .,{ }^{91}$ the fact that the semiempirical potential predicted the $\langle 110\rangle$ rather than the (correct) $\langle 111\rangle$ configuration as the lowest-energy defect structure makes it difficult to take the values found in Refs. 91 as reliable estimates for the diffusion coefficient of a self-interstitial atom defect.

The Debye temperature of tungsten $T_{D}$ is $310 \mathrm{~K}$. Hence, the statistics of thermal excitations at temperatures significantly lower than $T_{D}$ is dominated by quantum-mechanical effects, which are not adequately reproduced in a classical molecular dynamics simulation. The first of the three intervals of temperatures that we identified on the $D(T)$ curve shown in Fig. 15 lies entirely below $T_{D}$. Hence, the predicted behavior of the diffusion coefficient in this temperature range is unreliable. On the other hand, the part of the curve corresponding to $T \geqslant T_{D}$ is classical; hence, we can safely apply a classical analysis to the interpretation of simulations.

For $T>T_{D}$, we neglect the presence of the Peierls potential and assume that a defect performs classical Brownian motion described by the one-dimensional Langevin equation $^{92}$

$$
m^{*} \frac{d v}{d t}=-\gamma v(t)+f(t)
$$

Here, we take that rotations of the axis of the defect can be neglected, and $v(t)$ is the projection of the velocity of the defect on the direction of its axis. $m^{*}$ is the effective mass of the defect and $\gamma$ is the coefficient of dissipative friction. The stochastic thermal force $f(t)$ in the right-hand side of Eq. (17) has zero mean $\overline{f(t)}=0$. The solution of Eq. (17) has the form

$$
v(t)=\frac{1}{m^{*}} \int_{-\infty}^{t} d \tau \exp \left[-\frac{\gamma}{m^{*}}(t-\tau)\right] f(\tau) .
$$

Using this equation, we find the average kinetic energy of a migrating defect 


$$
\begin{aligned}
\frac{m^{*} \overline{v^{2}(t)}}{2}= & \frac{1}{2 m^{*}} \int_{-\infty}^{t} d \tau \int_{-\infty}^{t} d \tau^{\prime} \exp \left[-\frac{\gamma}{m^{*}}(t-\tau)\right] \\
& \times \exp \left[-\frac{\gamma}{m^{*}}\left(t-\tau^{\prime}\right)\right] \overline{f(\tau) f\left(\tau^{\prime}\right)} .
\end{aligned}
$$

Since the correlation time of the stochastic thermal force is many times shorter than the correlation time $t_{\text {migr }} \sim m^{*} / \gamma$ of velocity of the migrating crowdion, in Eq. (19) we may assume that the thermal force is delta correlated,

$$
\overline{f(\tau) f\left(\tau^{\prime}\right)}=f^{2} \delta\left(\tau-\tau^{\prime}\right) .
$$

Applying the equipartition principle $m^{*} v^{2}(t) / 2=k_{B} T / 2$, from Eqs. (19) and (20) we find that

$$
m^{*} \overline{v^{2}(t)} / 2=k_{B} T / 2=\frac{f^{2}}{2 m^{*}} \int_{-\infty}^{t} d \tau \exp \left[-\frac{2 \gamma}{m^{*}}(t-\tau)\right]=\frac{f^{2}}{4 \gamma}
$$

and arrive at a condition relating the amplitude of stochastic fluctuations of the thermal force and the coefficient of dissipative friction $\gamma$, namely,

$$
f^{2}=2 \gamma k_{B} T \text {. }
$$

This relation constitutes a general example of the fluctuation-dissipation theorem. Extending this approach to the treatment of the position of the defect $x(t)=\int_{-\infty}^{t} v(\tau) d \tau$, and defining the coefficient of one-dimensional diffusion as

$$
D=\lim _{t \rightarrow \infty} \overline{x^{2}(t)} / 2 t,
$$

we find that the coefficient of dissipative friction $\gamma$ and the diffusion coefficient are related by

$$
\gamma=\frac{k_{B} T}{D} .
$$

Using formula (23) and the data shown in Fig. 15, we find how the coefficient of dissipative friction of the crowdion defect varies as a function of absolute temperature. Figure 16 shows the friction coefficient $\gamma(T)$ plotted as a function of absolute temperature using the data for the diffusion coefficient $D(T)$ taken from Fig. 15. Figure 16 shows that in the range of relatively high temperatures $T \geqslant T_{D}$ (i.e., in the range of temperatures where the Brownian motion of crowdions can actually be approximated by the classical Langevin equation [Eq. (17)]), the effective friction coefficient is almost independent of absolute temperature. This agrees with molecular dynamics simulations performed by Zepeda-Ruiz et al. ${ }^{38}$ who also found that the diffusion coefficient was well approximated by a linear function of absolute temperature, implying that the friction coefficient was independent of $T$.

The finding that the thermal friction coefficient is independent of temperature is not obvious. Indeed, a single crowdion defect represents a limiting case of a small $\langle 111\rangle$ prismatic edge dislocation loop (see, e.g., Refs. 35, 39, 42, and 93), and hence it may seem that there should be no conceptual difference between the meaning of the coefficient of dissipative friction $\gamma$ entering Eq. (17) and the meaning of

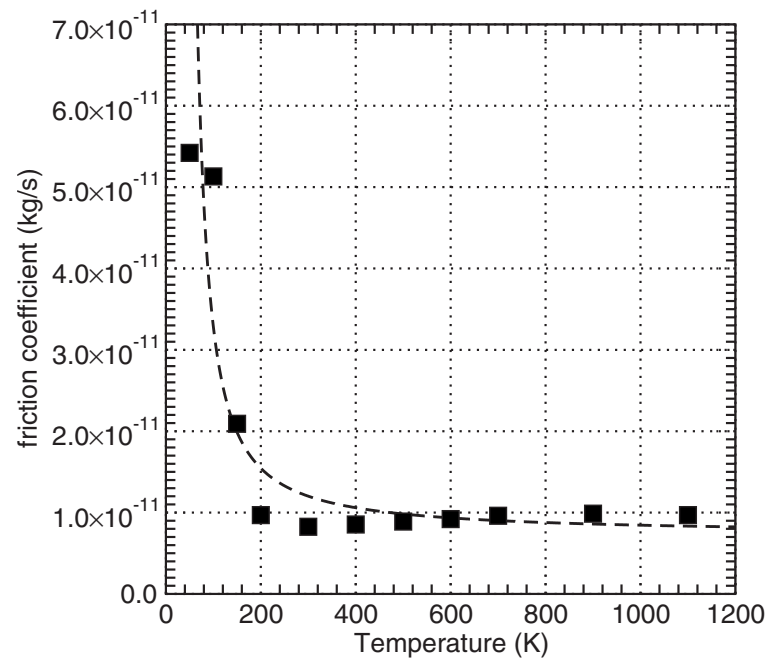

FIG. 16. The coefficient of dissipative thermal friction of a crowdion $\gamma=k_{B} T / D$ plotted as a function of absolute temperature. The dashed curve corresponds to the analytical formula for the diffusion coefficient shown by the dashed line in Fig. 15. The coefficient of thermal friction is almost independent of temperature for $T \geqslant T_{D}$, where thermal displacements of atoms in the lattice can be approximately treated as uncorrelated.

the coefficient of phonon drag $B$ describing the resistive force acting on a moving edge dislocation. For example, if instead of a fluctuating random force we introduce a timeindependent quantity $[f(t)=f]$ in the right-hand side of Eq. (17), the stationary solution will take the form

$$
v(t)=f / \gamma,
$$

similar to a solution for a uniformly moving dislocation.

In the interval of temperatures $T \geqslant T_{D}$, the phonon drag coefficient $B_{\mathrm{ph}}$ characterizing the motion of an edge dislocation in a material, according to calculations, varies linearly as a function of absolute temperature. ${ }^{94,95}$ The fact that the temperature dependence of the friction coefficient found in our simulations $(\gamma \approx$ const $)$ differs from that found in simulations of a moving edge dislocation, ${ }^{94,95}$ is interesting and requires further analysis. One possible line of reasoning that may explain the observed differences uses the fact that a linear edge dislocation forms a part an infinitely large dislocation loop and, hence, has an infinitely large effective mass. ${ }^{96} \mathrm{We}$ will not investigate this issue further in this paper. Instead, we well develop a microscopic analytically tractable model that will rationalize the behavior of the thermal friction coefficient of migrating crowdions found in our simulations

\section{FRENKEL-KONTOROVA MODEL FOR DISSIPATIVE FRICTION}

How do we evaluate the coefficient of dissipative friction for the Brownian motion of a crowdion in a crystal lattice at a finite temperature? To answer this question, we first define the notion of a crowdion as an object, characterized by its position in the crystal lattice. We then derive an equation of motion for the crowdion, taking into account the interaction between the defect and its thermal environment, and investi- 
gate solutions of this equation. We address the problem using the multistring Frenkel-Kontorova model. ${ }^{35}$ In this model, the defect is treated as a linear structure characterized by the orientation of its axis. The entire crystal lattice of atoms is described as an ensemble of linear strings of atoms running in the direction parallel to the axis of the crowdion. For example, the bcc lattice can be represented exactly as an ensemble of atomic strings running parallel to the [111] direction. The distance between neighboring atoms in each string is $a=\sqrt{3} a_{0} / 2$, where $a_{0}$ is the lattice constant, and in a plane normal to the [111] direction the atomic strings form a staggered hexagonal lattice. The position of an atom in the lattice is defined by two indices, namely, a two-dimensional vector index of a string $\mathbf{j}$ and the index of an atom in the string $n$. The Lagrangian of the model defined in terms of displacements $u_{\mathbf{j}, n}(t)$ of atoms in the direction of the strings has the form

$$
\begin{aligned}
\mathcal{L}= & \sum_{\mathbf{j}} \sum_{n=-\infty}^{\infty}\left\{\frac{m \dot{u}_{\mathbf{j}, n}(t)}{2}-\frac{\alpha}{2}\left[u_{\mathbf{j}, n+1}(t)-u_{\mathbf{j}, n}(t)\right]^{2}\right\} \\
& -\frac{m \omega^{2} a^{2}}{2 \pi^{2}} \sum_{\mathbf{j}, \mathbf{h}} \sum_{n=-\infty}^{\infty} \sin ^{2}\left[\frac{\pi\left(u_{\mathbf{j}, n}-u_{\mathbf{j}+\mathbf{h}, n}\right)}{a}\right],
\end{aligned}
$$

where $m$ is the mass of an atom, $\alpha$ is the parameter of elastic interaction between nearest-neighbor atoms belonging to the same string, and $\omega^{2}$ characterizes the strength of interaction between atoms belonging to adjacent strings. In other words, $\alpha$ gives the strength of the bond stretching term while $\omega^{2}$ is a parameter characterizing the magnitude of the bond bending term, i.e., the term associated with the displacement of adjacent atomic strings relative to each other. The Lagrangian equations of motion

$$
\frac{d}{d t} \frac{\partial \mathcal{L}}{\partial \dot{u}_{\mathbf{j}, n}(t)}=\frac{\partial \mathcal{L}}{\partial u_{\mathbf{j}, n}(t)}
$$

have the form

$$
\begin{aligned}
m \frac{d^{2} u_{\mathbf{j}, n}}{d t^{2}}= & \alpha\left(u_{\mathbf{j}, n+1}+u_{\mathbf{j}, n-1}-2 u_{\mathbf{j}, n}\right) \\
& -\frac{m \omega^{2} a}{\pi} \sum_{\mathbf{h}} \sin \left[\frac{2 \pi\left(u_{\mathbf{j}, n}-u_{\mathbf{j}+\mathbf{h}, n}\right)}{a}\right] .
\end{aligned}
$$

The field of atomic displacements $u_{\mathbf{j}, n}(t)$ entering this equation consists of two terms: a regular term describing the field of a defect migrating in the lattice and the random field of phonon excitations. Since the defect migrates in the direction of its axis and remains in the same atomic string, we represent the field of displacements as

$$
u_{\mathbf{j}, n}(t)=\mathcal{U}_{\mathbf{j}}[n-\mathcal{Z}(t) / a]+\Phi_{\mathbf{j}, n}(t),
$$

where $\mathcal{Z}(t)$ is the time-dependent position of the center of the defect in the lattice. Here, we assume that the strain field of the defect is translated coherently together with the core of the defect. The motion of the defect is assumed to be nonrelativistic; i.e., the velocity of the center of the defect $\dot{\mathcal{Z}}(t)$ is small in comparison to the speed of sound in the material. $\Phi_{\mathbf{j}, n}(t)$ denotes the field of phonon excitations.

The time derivatives of the displacement field of the defect have the form

$$
\begin{aligned}
\frac{d}{d t} \mathcal{U}_{\mathbf{j}}[n-\mathcal{Z}(t) / a]= & \mathcal{U}_{\mathbf{j}}^{\prime}[n-\mathcal{Z}(t) / a]\left[-\frac{1}{a} \frac{d \mathcal{Z}(t)}{d t}\right], \\
\frac{d^{2}}{d t^{2}} \mathcal{U}_{\mathbf{j}}[n-\mathcal{Z}(t) / a]= & -\mathcal{U}_{\mathbf{j}}^{\prime}[n-\mathcal{Z}(t) / a] \frac{1}{a} \frac{d^{2} \mathcal{Z}(t)}{d t^{2}} \\
& +\mathcal{U}_{\mathbf{j}}^{\prime \prime}[n-\mathcal{Z}(t) / a] \frac{1}{a^{2}}\left[\frac{d \mathcal{Z}(t)}{d t}\right]^{2} .
\end{aligned}
$$

Substituting these expressions into Eq. (25), we find

$$
\begin{gathered}
\left.\frac{m}{a^{2}}\left[\mathcal{Z}^{\prime}(t)\right]^{2} \mathcal{U}_{\mathbf{j}}^{\prime \prime}[n-\mathcal{Z}(t) / a]-\frac{m}{a}\left[\mathcal{Z}^{\prime \prime}(t)\right] \mathcal{U}_{\mathbf{j}}^{\prime}[n-\mathcal{Z}(t) / a]+m \frac{d^{2}}{d t^{2}} \Phi_{\mathbf{j}, n}(t)=\alpha \mathcal{U}_{\mathbf{j}}^{\prime \prime}[n-\mathcal{Z}(t) / a]\right)+\alpha\left[\Phi_{\mathbf{j}, n+1}(t)+\Phi_{\mathbf{j}, n-1}(t)-2 \Phi_{\mathbf{j}, n}(t)\right] \\
\quad-\frac{m \omega^{2} a}{\pi} \sum_{\mathbf{h}} \sin \left\{\frac{2 \pi}{a}\left[\mathcal{U}_{\mathbf{j}}[n-\mathcal{Z}(t) / a]+\Phi_{\mathbf{j}, n}(t)-\mathcal{U}_{\mathbf{j}+\mathbf{h}}[n-\mathcal{Z}(t) / a]-\Phi_{\mathbf{j}+\mathbf{h}, n}(t)\right]\right\}
\end{gathered}
$$

In the absence of the defect, the phonon displacements satisfy the equations

$$
\begin{aligned}
m \frac{d^{2} \Phi_{\mathbf{j}, n}}{d t^{2}}= & \alpha\left(\Phi_{\mathbf{j}, n+1}+\Phi_{\mathbf{j}, n-1}-2 \Phi_{\mathbf{j}, n}\right) \\
& -2 m \omega^{2} \sum_{\mathbf{h}}\left(\Phi_{\mathbf{j}, n}-\Phi_{\mathbf{j}+\mathbf{h}, n}\right)
\end{aligned}
$$

On the other hand, in the absence of phonons, the field of displacements of the defect satisfies the equation

$$
\begin{aligned}
\alpha \mathcal{U}_{\mathbf{j}}^{\prime \prime}[n-\mathcal{Z}(t) / a]= & \frac{m \omega^{2} a}{\pi} \sum_{\mathbf{h}} \sin \left\{\frac { 2 \pi } { a } \left[\mathcal{U}_{\mathbf{j}}[n-\mathcal{Z}(t) / a]\right.\right. \\
& \left.\left.-\mathcal{U}_{\mathbf{j}+\mathbf{h}}[n-\mathcal{Z}(t) / a]\right]\right\} .
\end{aligned}
$$

Equation (30) is valid for an arbitrary position of the center of the defect $\mathcal{Z}(t)$. Expanding the last term in Eq. (28) in the 
Taylor series, performing the summation over $n$, and taking into account the sum rules,

$$
\begin{aligned}
\sum_{n=-\infty}^{\infty} \mathcal{U}_{\mathbf{j}}^{\prime}[n-\mathcal{Z}(t) / a] & =\int_{-\infty}^{\infty} \mathcal{U}_{\mathbf{j}}^{\prime \prime}[n-\mathcal{Z}(t) / a] d n \\
& =\mathcal{U}_{\mathbf{j}}^{\prime}(\infty)-\mathcal{U}_{\mathbf{j}}^{\prime}(-\infty)=0, \\
\sum_{n=-\infty}^{\infty} \mathcal{U}_{\mathbf{j}}^{\prime}[n-\mathcal{Z}(t) / a]= & \int_{-\infty}^{\infty} \mathcal{U}_{\mathbf{j}}^{\prime}[n-\mathcal{Z}(t) / a] d n \\
& =\mathcal{U}_{\mathbf{j}}(\infty)-\mathcal{U}_{\mathbf{j}}(-\infty)=-a \delta_{\mathbf{j}, 0},
\end{aligned}
$$

we arrive at the equation of motion for the crowdion defect

$$
m \frac{d^{2} \mathcal{Z}}{d t^{2}}=4 m \omega^{2} \sum_{n, \mathbf{h}}\left[\Phi_{n, 0}(t)-\Phi_{n, \mathbf{h}}(t)\right] \sin ^{2}\left\{\frac{\pi}{a} \mathcal{U}_{0}[n-\mathcal{Z}(t) / a]\right\} .
$$

Since $\mathcal{U}_{0}[n-\mathcal{Z}(t) / a]$ describes the field of displacements in the central string of the crowdion defect, we approximate it by the soliton solution of the single-string FrenkelKontorova model, ${ }^{35}$

$$
\mathcal{U}_{0}[n-\mathcal{Z}(t) / a]=\frac{2 a}{\pi} \arctan \left\{\exp \left[\frac{n a-\mathcal{Z}(t)}{\mathcal{N} a}\right]\right\},
$$

where $\mathcal{N}$ is the dimensionless width of the crowdion solution. Formula (33) approximates the field of atomic displacements in the crowdion defect very well, compared to the DFT and empirical potential values. ${ }^{35}$

By substituting Eq. (33) into Eq. (32), we arrive at

$$
\frac{d^{2} \mathcal{Z}}{d t^{2}}=4 \omega^{2} \sum_{n, \mathbf{h}} \frac{\Phi_{n, 0}(t)-\Phi_{n, \mathbf{h}}(t)}{\cosh ^{2}\left\{\left[n-\frac{\mathcal{Z}(t)}{a}\right] / \mathcal{N}\right\}} .
$$

This simple and compact equation describes the effect of stochastic thermal displacements of atoms on the onedimensional motion of a crowdion in the lattice.

Equation (34) does not include the effect of rotations of the axis of the crowdion on its migration in the lattice. The mathematical formalism that makes it possible to include the effect of rotation of the direction of migration of a crowdion in the treatment of diffusion of defects was developed in Ref. 97.

The right-hand side of Eq. (34), multiplied by the effective mass of the defect, gives the stochastic force acting on the crowdion

$$
f(t)=4 m^{*} \omega^{2} \sum_{n, \mathbf{h}} \frac{\Phi_{n, 0}(t)-\Phi_{n, \mathbf{h}}(t)}{\cosh ^{2}\left\{\left[n-\frac{\mathcal{Z}(t)}{a}\right] / \mathcal{N}\right\}} .
$$

This equation shows that it is the difference between phonon displacements of atoms in adjacent strings that gives rise to the force randomly accelerating or decelerating the defect. Phonon displacements of atoms in the same string have much weaker effect on the motion of the crowdion than the relative displacements of two neighbouring strings. Also, Eq. (35) shows that only phonon displacements of atoms near the core of the defect make a significant contribution to the stochastic force driving the Brownian motion of the defect.

The mean value of $f(t)$ given by Eq. (35) is zero $\overline{f(t)}=0$, and the correlation function of fluctuations of this force is approximately given by

$$
\overline{f(t) f\left(t^{\prime}\right)}=16\left(m^{*}\right)^{2} \omega^{4} \sum_{n, \mathbf{h}} \frac{\overline{\Phi_{n, 0}(t) \Phi_{n, 0}\left(t^{\prime}\right)}+\overline{\Phi_{n, \mathbf{h}}(t) \Phi_{n, \mathbf{h}}\left(t^{\prime}\right)}}{\cosh ^{4}\left\{\left[n-\frac{\mathcal{Z}(t)}{a}\right] / \mathcal{N}\right\}} .
$$

In this equation, we neglected correlations of thermal displacements of different atoms in the lattice. This approximation works well in the high-temperature limit $T \geqslant T_{D}$ and is equivalent to the Einstein model of thermal displacements. Since in the bcc lattice the statistics of thermal displacements of atoms is independent of the lattice site, Eq. (36) can be further simplified as

$$
\overline{f(t) f\left(t^{\prime}\right)}=256\left(m^{*}\right)^{2} \omega^{4} \mathcal{N} \overline{\Phi(t) \Phi\left(t^{\prime}\right)} .
$$

Here, we used the formula

$$
\int_{-\infty}^{\infty} \frac{d n}{\cosh ^{4}(n / \mathcal{N})}=4 \mathcal{N} / 3
$$

and the fact that each $\langle 111\rangle$ string of atoms is surrounded by six neighboring strings. The correlation function of thermal displacements of atoms in the limit of high temperatures has the form

$$
\overline{\Phi(t) \Phi\left(t^{\prime}\right)}=\frac{k_{B} T}{m \omega_{D}^{2}} \overline{\cos \left[\omega_{D}\left(t-t^{\prime}\right)\right]} \approx \frac{k_{B} T}{m \omega_{D}^{3}} \delta\left(t-t^{\prime}\right) .
$$

Comparing Eqs. (37) and (38) with Eq. (22), we arrive at an analytical estimate for the coefficient of thermal friction

$$
\gamma=256 \frac{\left(m^{*}\right)^{2} \omega^{4} \mathcal{N}}{m \omega_{D}^{3}} .
$$

Substituting numerical values of parameters in this formula, we find that the magnitude of the friction coefficient is of the order of $10^{-11} \mathrm{~kg} / \mathrm{s}$. This estimate is close to the values found using molecular dynamics simulations in the limit $T$ $\geqslant T_{D}$. What is even more significant is that, according to Eq. (39), in the classical limit $T \geqslant T_{D}$ the coefficient of thermal friction is independent of absolute temperature, and the diffusion coefficient $D(T)$ varies linearly as a function of absolute temperature, $D(T) \sim T$. This conclusion agrees with molecular dynamics simulations of the Brownian motion of crowdions described in this paper, and it also agrees with earlier studies of migration of crowdions in vanadium carried out in Ref. 38. 
TABLE III. Group V empirical potentials.

\begin{tabular}{|c|c|c|c|c|c|}
\hline \multicolumn{2}{|c|}{ Vanadium } & \multicolumn{2}{|c|}{ Niobium } & \multicolumn{2}{|c|}{ Tantalum } \\
\hline$A$ & 5.439922189462638 & $A$ & 9.292960445740691 & $A$ & 10.01248520934047 \\
\hline$r_{n}^{f}$ & $f_{n}$ & $r_{n}^{f}$ & $f_{n}$ & $r_{n}^{f}$ & $f_{n}$ \\
\hline 3.190000000000000 & 1.890275993289541 & 3.463800000000000 & 1.315820563897800 & 3.469100000000000 & 1.411466249903413 \\
\hline 3.040000000000000 & -2.575125966545582 & 3.300933333333333 & -1.553583145488597 & 3.305966666666666 & -1.742457130439156 \\
\hline 2.890000000000000 & -2.333619727690528 & 3.138066666666667 & -1.863852095850426 & 3.142833333333333 & -2.163310282516732 \\
\hline 2.740000000000000 & 5.468040512088823 & 2.975200000000000 & 2.642548215724037 & 2.979700000000000 & 3.122206593519948 \\
\hline 2.632600000000000 & -0.9366477843821801 & 2.858500000000000 & -0.1538514822429389 & 2.862900000000000 & $.8873075878678840 \times 10^{-2}$ \\
\hline 2.500970000000000 & 1.880297597456256 & 2.719755000000000 & -1.088525289528290 & 2.719755000000000 & -0.2086354380181568 \\
\hline 2.369340000000000 & 0.6100939173059921 & 2.572650000000000 & 8.448251111154777 & 2.576610000000000 & 0.6939195820784342 \\
\hline$r_{n}^{V}$ & $V_{n}$ & $r_{n}^{V}$ & $V_{n}$ & $r_{n}^{V}$ & $V_{n}$ \\
\hline 4.100000000000000 & -0.9989201728470961 & 4.451900000000000 & -0.9712655459247255 & 4.458600000000000 & -1.010535357881566 \\
\hline 3.828000000000000 & 2.508315506019401 & 4.156560000000000 & 2.546718802733285 & 4.162820000000000 & 2.905987065650308 \\
\hline 3.556000000000000 & -3.762034795839844 & 3.861220000000000 & -3.877555625741159 & 3.867040000000000 & -5.053535078225670 \\
\hline 3.284000000000000 & 11.30374353457620 & 3.565880000000000 & 12.49074179239876 & 3.571260000000000 & 15.11704171653469 \\
\hline 3.012000000000000 & -19.12920476121472 & 3.270540000000000 & -21.25877974460241 & 3.275480000000000 & -26.10466457022816 \\
\hline 2.740000000000000 & 18.33316600617811 & 2.975200000000000 & 10.47543658678679 & 2.979700000000000 & 15.45408459383589 \\
\hline 2.632600000000000 & 4.485535455224519 & 2.858500000000000 & 17.73776069701092 & 2.862900000000000 & 12.48335338054882 \\
\hline 2.500970000000000 & -0.3765517276879735 & 2.719755000000000 & -36.10313653693880 & 2.719755000000000 & -8.751933980136855 \\
\hline 2.369340000000000 & 1.148339795860195 & 2.572650000000000 & 90.15287081731522 & 2.576610000000000 & 4.580222062018542 \\
\hline
\end{tabular}

\section{SUMMARY}

In this paper, we presented a density-functional theory study of the electronic structure of self-interstitial atom and vacancy defects in all the bcc transition metals, developed parametrized forms of the Finnis-Sinclair many-body potentials for five of those metals, and investigated the statistics of

TABLE IV. Group VI empirical potentials.

\begin{tabular}{|c|c|c|c|}
\hline \multicolumn{2}{|c|}{ Molybdenum } & \multicolumn{2}{|c|}{ Tungsten } \\
\hline$A$ & 7.709236888883772 & $A$ & 10.84238200368439 \\
\hline$r_{n}^{f}$ & $f_{n}$ & $r_{n}^{f}$ & $f_{n}$ \\
\hline 3.302700000000000 & 1.601365370271091 & 3.321600000000000 & 1.677334871575606 \\
\hline 3.147366666666667 & -1.627314671935321 & 3.165333333333333 & -1.673137509507131 \\
\hline 2.992033333333334 & -3.873039838840650 & 3.009066666666667 & -4.737051994974169 \\
\hline 2.836700000000000 & 5.863098272130567 & 2.852800000000000 & 7.870789420780674 \\
\hline 2.725500000000000 & 1.062058443655675 & 2.741100000000000 & $-3.7924230525563378 \times 10^{-4}$ \\
\hline \multirow[t]{2}{*}{2.589225000000000} & 2.690089456866705 & 2.604045000000000 & 6.190816158916646 \\
\hline & & 2.466990000000000 & -0.9565713891199151 \\
\hline$r_{n}^{V}$ & $V_{n}$ & $r_{n}^{V}$ & $V_{n}$ \\
\hline 4.244700000000000 & -0.6022171729570738 & 4.268900000000000 & -0.1036435865158945 \\
\hline 3.963100000000000 & 1.637778152210674 & 3.985680000000000 & -0.2912948318493851 \\
\hline 3.681500000000000 & -4.443742707968704 & 3.702460000000000 & -2.096765499656263 \\
\hline 3.399900000000000 & 16.37678840233729 & 3.419240000000000 & 19.16045452701010 \\
\hline 3.118300000000000 & -28.09191863719095 & 3.136020000000000 & -41.01619862085917 \\
\hline 2.836700000000000 & 22.82129576675600 & 2.852800000000000 & 46.05205617244703 \\
\hline 2.725500000000000 & 21.13598549564144 & 2.741100000000000 & 26.42203930654883 \\
\hline \multirow[t]{2}{*}{2.589225000000000} & 9.417069069515332 & 2.604045000000000 & 15.35211507804088 \\
\hline & & 2.466990000000000 & 14.12806259323987 \\
\hline
\end{tabular}


the thermally activated migration of self-interstitial atom crowdion defects in tungsten. By investigating the logical connection between the electronic, atomistic, and mesoscopic aspects of the problem, we highlighted the pivotal role played by the electronic structure of defects, the use of controlled approximations in deriving the semiempirical potentials, and the essential part played by solvable analytical models in rationalizing the information provided by atomistic simulations. In all the bcc metals except Fe, a selfinterstitial atom defect adopts a linear $\langle 111\rangle$ crowdion configuration, which at a finite temperature migrates in the direction of its axis, occasionally switching between equivalent crystallographic orientations. The diffusion coefficient of crowdions found in our simulations for tungsten is many orders of magnitude higher than the diffusion coefficient found for the same metal in earlier simulations, where the semiempirical interatomic potential did not agree with the formation energies of point defects predicted by densityfunctional theory. This highlights the significance of the parametrization of the semiempirical potentials given in this paper. We expect that the potentials, constructed in such a way as to be able to retain a sufficiently high accuracy over a range of strongly distorted atomistic configurations, will help understand the nature of atomistic processes responsible for the changes in microstructure occurring in materials under irradiation.

\section{ACKNOWLEDGMENTS}

We would like to thank R. Bullough, C.-H. Woo, W. Frank, J. H. Evans, A.P. Horsfield, P. Olsson, M. Victoria, and J.-L. Boutard for stimulating discussions, and T. Martin for his kind help with using the parallel computing facilities at UKAEA Culham Science Centre. Work at UKAEA was supported by the UK Engineering and Physical Sciences Research Council, by EURATOM, and by EXTREMAT integrated project under Contract No. NMP3-CT-2004-500253.

\section{APPENDIX: EMPIRICAL POTENTIAL PARAMETRIZATIONS}

Tables III and IV contain the complete parametrization of the semiempirical interatomic potential for the five bcc nonmagnetic metals considered in this study. For accuracy, all digits printed in the tables should be employed. We remind the reader that these potentials are currently unsuitable for modeling high-energy collision dynamics involving, for example, the early stages of cascade simulation evolution.
${ }^{1}$ H. R. Paneth, Phys. Rev. 80, 708 (1950).

${ }^{2}$ W. M. Lomer and A. H. Cottrell, Philos. Mag. 46, 711 (1955).

${ }^{3}$ T. H. Blewitt, R. R. Coltman, C. E. Klabunde, and T. S. Noggle, J. Appl. Phys. 28, 639 (1957).

${ }^{4}$ L. Tewordt, Phys. Rev. 109, 61 (1958).

${ }^{5}$ F. W. de Wette, Phys. Rev. 135, A287 (1964).

${ }^{6}$ W. Frank and A. Seeger, Radiat. Eff. 1, 117 (1969).

${ }^{7}$ A. Seeger and K. P. Chik, Phys. Status Solidi 29, 455 (1968).

${ }^{8}$ A. F. W. Willoughby, Rep. Prog. Phys. 41, 1665 (1978).

${ }^{9}$ R. Car, P. J. Kelly, A. Oshiyama, and S. T. Pantelides, Phys. Rev. Lett. 52, 1814 (1984).

${ }^{10}$ A. Seeger, Phys. Status Solidi 38, 235 (1970).

${ }^{11}$ W. Schilling, K. Schroeder, and H. Wollenberger, Phys. Status Solidi 38, 245 (1970).

${ }^{12}$ W. Frank, Radiat. Eff. Lett. Sect. 86, 107 (1984).

${ }^{13}$ W. Frank and A. Seeger, Mater. Sci. Forum 15-18, 57 (1987).

${ }^{14}$ C. H. Woo and W. Frank, J. Nucl. Mater. 137, 7 (1985); 140, 214 (1986); Mater. Sci. Forum 15-18, 875 (1987).

${ }^{15}$ V. L. Indenbom, Zh. Eksp. Teor. Fiz. 12, 526 (1970) [Sov. Phys. JETP 12, 369 (1970)].

${ }^{16}$ Zh. I. Dranova, A. M. Dyachenko, and I. M. Mikhailovskii, Zh. Eksp. Teor. Fiz. 13, 40 (1971) [Sov. Phys. JETP 13, 27 (1970)].

${ }^{17}$ I. M. Mikhailovskii and Zh. I. Dranova, Zh. Eksp. Teor. Fiz. 63, 567 (1972) [Sov. Phys. JETP 36, 300 (1973)].

${ }^{18}$ F. Dausinger and H. Schultz, Phys. Rev. Lett. 35, 1773 (1975).

${ }^{19}$ H. Schultz, Mater. Sci. Eng., A 141, 149 (1991).

${ }^{20}$ Atomic Defects in Metals, Landolt-Börnstein New Series, Group III, Vol. 25, edited by H. Ullmaier (Springer-Verlag, Berlin, 1991).

${ }^{21}$ P. Ehrhart, J. Nucl. Mater. 69/70, 200 (1978).

${ }^{22}$ G. J. Ackland and R. Thetford, Philos. Mag. A 56, 15 (1987).
${ }^{23}$ W. Xu and J. A. Moriarty, Phys. Rev. B 54, 6941 (1996).

${ }^{24}$ M. H. Carlberg, E. P. Münger, and L. Hultman, J. Phys.: Condens. Matter 11, 6509 (1999); 12, 79 (2000).

${ }^{25}$ G. Simonelli, R. Pasianot, and E. J. Savino, Phys. Status Solidi B 217, 747 (2000).

${ }^{26}$ J. A. Moriarty, J. F. Belak, R. E. Rudd, P. Söderlind, F. H. Streitz, and L. H. Yang, J. Phys.: Condens. Matter 14, 2825 (2002).

${ }^{27}$ C. Domain and C. S. Becquart, Phys. Rev. B 65, 024103 (2001).

${ }^{28}$ C.-C. Fu, F. Willaime, and P. Ordejón, Phys. Rev. Lett. 92, 175503 (2004).

${ }^{29}$ F. Willaime, C.-C. Fu, M. C. Marinica, and J. Dalla Torre, Nucl. Instrum. Methods Phys. Res. B 228, 92 (2005).

${ }^{30}$ S. Han, L. A. Zepeda-Ruiz, G. J. Ackland, R. Car, and D. J. Srolovitz, Phys. Rev. B 66, 220101(R) (2002).

${ }^{31}$ D. Nguyen-Manh, A. P. Horsfield, and S. L. Dudarev, Phys. Rev. B 73, 020101(R) (2006).

${ }^{32}$ A. M. Kossevich, The Crystal Lattice (Wiley-VCH, Berlin, 1999).

${ }^{33}$ A. S. Kovalev, A. D. Kondratyuk, A. M. Kosevich, and A. I. Landau, Phys. Rev. B 48, 4122 (1993).

${ }^{34}$ V. D. Natsik and Y. I. Nazarenko, Eur. Phys. J. B 29, 285 (2002).

${ }^{35}$ S. L. Dudarev, Philos. Mag. 83, 3577 (2003).

${ }^{36}$ O. M. Braun and Yu. S. Kivshar, Phys. Rep. 306, 1 (1998).

${ }^{37}$ O. V. Charkina and M. M. Bogdan, Symmetry, Integr. Geom.: Methods Appl. 2, 047 (2006).

${ }^{38}$ L. A. Zepeda-Ruiz, J. Rottler, S. Han, G. J. Ackland, R. Car, and D. J. Srolovitz, Phys. Rev. B 70, 060102(R) (2004); L. A. Zepeda-Ruiz, J. Rottler, B. D. Wirth, R. Car, and D. J. Srolovitz, Acta Mater. 53, 1985 (2005).

${ }^{39}$ B. D. Wirth, G. R. Odette, D. Maroudas, and G. E. Lucas, J. Nucl. Mater. 244, 185 (1997). 
${ }^{40}$ B. D. Wirth, G. R. Odette, D. Maroudas, and G. E. Lucas, J. Nucl. Mater. 276, 33 (2000).

${ }^{41}$ Yu. N. Osetsky, D. J. Bacon, A. Serra, B. N. Singh, and S. I. Golubov, J. Nucl. Mater. 276, 65 (2000); Philos. Mag. 83, 61 (2003).

${ }^{42}$ Y. N. Osetsky, A. Serra, B. N. Singh, and S. I. Golubov, Philos. Mag. A 80, 2131 (2000).

${ }^{43}$ A. V. Barashev, Yu. N. Osetsky, and D. J. Bacon, Philos. Mag. A 80, 2709 (2000).

${ }^{44}$ E. Kuramoto, K. Ohsawa, and T. Tsutsumi, Mater. Trans., JIM 46, 450 (2005).

${ }^{45}$ J. Marian, B. D. Wirth, and J. M. Perlado, Phys. Rev. Lett. 88, 255507 (2002).

${ }^{46}$ S. D. Kenny, A. P. Horsfield, and H. Fujitani, Phys. Rev. B 62 , 4899 (2000).

${ }^{47}$ K. Kohary, V. M. Burlakov, D. G. Pettifor, and D. Nguyen-Manh, Phys. Rev. B 71, 184203 (2005).

${ }^{48}$ C. Hartwigsen, S. Goedecker, and J. Hutter, Phys. Rev. B 58, 3641 (1998).

${ }^{49}$ J. P. Perdew, K. Burke, and M. Ernzerhof, Phys. Rev. Lett. 77, 3865 (1996).

${ }^{50}$ D. A. Pankhurst, D. Nguyen-Manh, and D. G. Pettifor, Phys. Rev. B 69, 075113 (2004)

${ }^{51}$ G. Kresse and J. Hafner, Phys. Rev. B 47, 558 (1993).

${ }^{52}$ G. Kresse and J. Furthmuller, Phys. Rev. B 54, 11169 (1996).

${ }^{53}$ G. Kresse and J. Hafner, Comput. Mater. Sci. 6, 15 (1996).

${ }^{54}$ D. Nguyen-Manh, V. Vitek, and A. P. Horsfield, Prog. Mater. Sci. 52, 255 (2007).

${ }^{55}$ D. Nguyen-Manh, S. L. Dudarev, and A. P. Horsfield, J. Nucl. Mater. 367-370, 257 (2007).

${ }^{56}$ C. Kittel, Introduction to Solid State Physics, 5th. ed. (Wiley, NewYork, 1976).

${ }^{57}$ B. Zhang, Y. Ouyang, S. Liao, and Z. Jin, Physica B 262, 218 (1999).

${ }^{58}$ K. Masuda, in Proceedings of the Yamada Conference on Point Defects and Defect Interactions in Metals, edited by J. Takamura, M. Doyama, and M. Kiritani (University of Tokyo Press, Tokyo, 1982), p. 105

${ }^{59}$ P. Olsson, Ph.D. thesis, Uppsala University, 2005; P. Olsson, J. Wallenius, C. Domain, K. Nordlund, and L. Malerba, Phys. Rev. B 72, 214119 (2005).

${ }^{60}$ C. J. Forst, J. Slycke, K. J. Van Vliet, and S. Yip, Phys. Rev. Lett. 96, 175501 (2006).

${ }^{61}$ J. P. Perdew and Y. Wang, Phys. Rev. B 45, 13244 (1991).

${ }^{62}$ H. Johnson, G. Mills, and K. W. Jacobsen, in Classical and Quantum Dynamics in Condensed Phase Simulations, edited by B. J. Berne et al. (World Scientific, Singapore, 1998), p. 385.

${ }^{63}$ T. R. Armstrong, H. A. Filius, A. van Veen, and J. R. Heringa, J. Nucl. Mater. 203, 189 (1993).

${ }^{64}$ K. Yamakawa, H. Kugler, and H. Schultz, Radiat. Eff. 105, 171 (1988).

${ }^{65}$ R. A. Johnson, in Diffusion in Body Centered Cubic Metals, edited by J. A. Wheeler and F. R. Winslow (American Society for Metals, Metals Park, OH, 1965), p. 357.

${ }^{66}$ See Ref. 20, p. 182; J. Y. Park, H. C. W. Huang, R. W. Siegel, and R. W. Balluffi, MIT Technical Report No. DOE/ER/05002-31, 1983 (unpublished).

${ }^{67}$ M. Eldrup, O. E. Mogensen, and J. H. Evans, J. Phys. F: Met.
Phys. 6, 499 (1976).

${ }^{68}$ D. Nguyen-Manh and S. L. Dudarev, Mater. Sci. Eng., A 423, 74 (2006).

${ }^{69}$ D. Nguyen-Manh and S. L. Dudarev, Comput. Mater. Sci. 36, 112 (2006).

${ }^{70}$ K. Arakawa, M. Hatanaka, E. Kuramoto, K. Ono, and H. Mori, Phys. Rev. Lett. 96, 125506 (2006).

${ }^{71}$ F. Willaime, A. Satta, M. Nastar, and O. Lebacq, Int. J. Quantum Chem. 77, 927 (2000).

${ }^{72}$ S. M. Lee, R. J. Nicholls, D. Nguyen-Manh, D. G. Pettifor, G. A. D. Briggs, S. Lazar, and D. J. H. Cockayne, Chem. Phys. Lett. 404, 206 (2005).

${ }^{73}$ R. J. Nicholls, D. Nguyen-Manh, and D. J. H. Cockayne, J. Phys.: Conf. Ser. 26, 153 (2006).

${ }^{74}$ A. Satta, F. Willaime, and S. de Gironcoli, Phys. Rev. B 57, 11184 (1998); 60, 7001 (1999).

${ }^{75}$ M. W. Finnis and J. E. Sinclair, Philos. Mag. A 50, 45 (1984).

${ }^{76}$ G. J. Ackland, M. W. Finnis, and V. Vitek, J. Phys. F: Met. Phys. 18, L153 (1988).

${ }^{77}$ S. L. Dudarev, J. Phys.: Condens. Matter 18, S447 (2006).

${ }^{78}$ N. W. Ashcroft and N. D. Mermin, Solid State Physics (Saunders College, Philadelphia, 1988), Chap. 25.

${ }^{79}$ A. P. Sutton, Philos. Mag. A 60, 146 (1989).

${ }^{80}$ M. T. Dove, Structure and Dynamics: An Atomic View of Materials (Oxford University Press, Oxford, 2003), Chap. 11.

${ }^{81}$ A. Corana, M. Marchesi, C. Martini, and S. Ridella, ACM Trans. Math. Softw. 13, 262 (1987).

${ }^{82}$ J. A. Nelder and R. Mead, Comput. J. 7, 308 (1965).

${ }^{83}$ S. L. Dudarev and P. M. Derlet, J. Phys.: Condens. Matter 17, 7097 (2005); P. M. Derlet and S. L. Dudarev, Prog. Mater. Sci. 52, 299 (2007).

${ }^{84}$ M. Gilbert (unpublished).

${ }^{85}$ M. W. Guinan, R. N. Stuart, and R. J. Borg, Phys. Rev. B 15, 699 (1977).

${ }^{86}$ R. M. Mazo, Brownian Motion: Fluctuations, Dynamics and Applications (Clarendon, Oxford, 2002).

${ }^{87}$ W. T. Coffey, Yu. P. Kalmykov, and T. T. Waldron, The Langevin Equation (World Scientific, Singapore, 2004).

${ }^{88}$ S. Chandrasekhar, Rev. Mod. Phys. 15, 1 (1943).

${ }^{89}$ N. Soneda and T. Diaz de la Rubia, Philos. Mag. A 78, 995 (1998).

${ }^{90}$ N. Soneda and T. Diaz de la Rubia, Philos. Mag. A 81, 331 (2001).

${ }^{91}$ M. H. Carlberg, E. P. Münger, and L. Hultman, J. Phys.: Condens. Matter 11, 6509 (1999); 12, 79 (2000).

${ }^{92}$ S. L. Dudarev, J. Nucl. Mater. 307-311, 881 (2002); Phys. Rev. B 65, 224105 (2002).

${ }^{93}$ J. Marian, B. D. Wirth, A. Caro, B. Sadigh, G. R. Odette, J. M. Perlado, and T. Diaz de la Rubia, Phys. Rev. B 65, 144102 (2002).

${ }^{94}$ G. Leibfried, Z. Phys. 127, 344 (1950).

${ }^{95}$ E. Bitzek and P. Gumbsch, Mater. Sci. Eng., A 387-389, 11 (2004).

${ }^{96}$ S. L. Dudarev, Comptes Rendus Acad. Sci. Paris, Ser. Physique (unpublished).

${ }^{97}$ T. S. Hudson, S. L. Dudarev, and A. P. Sutton, Proc. R. Soc. London, Ser. A 460, 2457 (2004). 